\title{
COMPOSICIÓN Y ASPECTOS ESTRUCTURALES DE LOS BOSQUES DE ENCINO DE LA SIERRA DE ZAPALINAMÉ, COAHUILA, MÉXICO
}

\author{
Juan Antonio Encina Domínguez 1 , Alejandro Zárate Lupercio 1 , Eduardo Estrada \\ CAstillón ${ }^{2}$, Jesús Valdés Reyna ${ }^{3}$ y José Ángel Villarreal Quintanilla ${ }^{3}$ \\ ${ }^{1}$ Universidad Autónoma Agraria Antonio Narro, Departamento Forestal, \\ 25315 Buenavista, Saltillo, Coahuila, México.juanencina@gmail.com \\ ${ }^{2}$ Universidad Autónoma de Nuevo León, Facultad de Ciencias Forestales, \\ Apdo. postal 41, 6700 Linares, Nuevo León, México. \\ ${ }^{3}$ Universidad Autónoma Agraria Antonio Narro, Departamento de Botánica, \\ 25315 Buenavista, Saltillo, Coahuila, México.
}

\section{RESUMEN}

Con la finalidad de conocer la estructura y composición florística de los bosques de encino de la Sierra de Zapalinamé, se evaluó la densidad y área basal del arbolado adulto, la cobertura de los arbustos y se registró la identidad de los componentes herbáceos en 66 parcelas de $500 \mathrm{~m}^{2}$. Los árboles juveniles se midieron en sitios de $50 \mathrm{~m}^{2}$ y la regeneración en cinco sitios de $1 \mathrm{~m}^{2}$. Los atributos de la vegetación fueron utilizados para obtener el valor de importancia ecológica para las especies leñosas. La flora vascular es de 259 especies y taxa infraespecíficos en 178 géneros y 67 familias. Las asociaciones vegetales definidas mediante el análisis de conglomerados son el bosque de Quercus greggii - Q. mexicana y de Quercus saltillensis - Q. laeta. Los encinos estructuralmente importantes son Quercus greggii (1480 ind ha-1), Q. saltillensis (876), Q. laeta (741) y Q. mexicana (284). La densidad total del arbolado adulto fue de $4448 \mathrm{ind} \mathrm{ha-}^{-1} \mathrm{y}$ fue mayor que la de renuevos $\left(269 \mathrm{ind} \mathrm{ha}^{-1}\right) \mathrm{e}$ individuos juveniles (175 ind ha ${ }^{-1}$. Los encinos con mayor área basal fueron Quercus greggii $\left(9.0 \mathrm{~m}^{2} \mathrm{ha}^{-1}\right)$ y $Q$. saltillensis $\left(6.62 \mathrm{~m}^{2} \mathrm{ha}^{-1}\right)$. De las 37 parejas interespecíficas de encinos analizadas, $62 \%$ se asociaron significativamente. Los arbustos con mayor cobertura fueron: Garrya glaberrima, Ageratina ligustrina y A. saltillensis. El estrato herbáceo representó $72.9 \%$ de la flora y sus elementos más comunes fueron Artemisia ludoviciana, Pleopeltis guttata, Cheilanthes tomentosa y Achillea millefolium. El número de especies decreció con el aumento de la altitud.

Palabras clave: área basal, Coahuila, densidad, disturbio antropogénico, México, Quercus, Sierra de Zapalinamé. 


\section{ABSTRACT}

To determine the structure and floristic composition of the oak forest of the Sierra de Zapalinamé, we established sixty six plots of $500 \mathrm{~m}^{2}$. Density and basal area were measured for adult trees and density and cover in shrubs. Saplings were considered in concentric areas of $50 \mathrm{~m}^{2}$ and seedlings in five areas of $1 \mathrm{~m}^{2}$. All the plants were collected and identified. Vegetation attributes were used to obtain the importance value for trees and shrubs. The vascular flora takes in 259 species and infraspecific taxa with 178 genera in 67 plant families. The forest associations were defined by Quercus greggii - Q. mexicana and Quercus saltillensis - Q. laeta; the most important oaks in the forest structure were Quercus greggii (1480 ind ha $\left.{ }^{-1}\right)$, Q. saltillensis (876), Q. laeta (741) and Q. mexicana (284). The mature tree density (4448 ind ha $\mathrm{a}^{-1}$ ) differs opposed to the seedlings (269 ind ha $\left.{ }^{-1}\right)$ and saplings (175 ind $\mathrm{ha}^{-1}$ ). The oaks with the highest basal area were Quercus greggii with $9.0 \mathrm{~m}^{2} \mathrm{ha}^{-1}$ and $Q$. saltillensis $6.62 \mathrm{~m}^{2} \mathrm{ha}^{-1}$. Thirty seven pairs of species were analyzed and $62 \%$ have significant association. The shrubs with the highest value of covering were: Garrya glaberrima, Ageratina ligustrina and A. saltillensis. The herbs were $72.9 \%$ of the flora and the most common elements were: Artemisia ludoviciana, Pleopeltis guttata, Cheilanthes tomentosa and Achillea millefolium. The species number diminishes when the altitude increases.

Key words: anthropogenic Coahuila, disturbance, basal area, density, Mexico, Quercus, Sierra de Zapalinamé.

\section{INTRODUCCIÓN}

Los bosques de encino son comunidades propias de las regiones templadas y subtropicales del hemisferio norte (Nixon, 1993), y en particular de las zonas montañosas de México (Rzedowski, 1978). Las montañas del centro y sur del país, además de la Sierra Madre Oriental, son las áreas con mayor diversidad de especies del género Quercus (Nixon, 1993). En comunidades boscosas la ocurrencia de incendios (Abrams, 1992; Zavala, 2000) y la utilización de la vegetación a través de apacentamiento (Quintana et al., 1992; Pettit et al., 1995) son factores que provocan cambios marcados en la composición florística y condicionan la estructura de la vegetación (Jardel, 1991; Attiwill, 1994; González et al., 1991, 1995a y 1995b). Tales cambios varían con el origen, extensión, régimen e intensidad del disturbio (Rzedowski, 1978; Spurr y Barnes, 1982; White y Pickett, 1985), así como con la respuesta de las especies de las comunidades vegetales (Hobbs y Huenneke, 1992). Como consecuencia de la influencia humana, existe una tendencia de los bosques a la sencillez estructural, coetaneidad, menor diversidad de especies y mayor susceptibilidad a las invasoras (Jardel, 1986, Hobbs y Huenneke, 1992). 
La Sierra de Zapalinamé es un área protegida bajo la categoría de Zona Sujeta a Conservación Ecológica (Anónimo, 1996), se ubica en el área transicional entre la Sierra Madre Oriental y el Desierto Chihuahuense (Anónimo, 1998). Sus bosques de encino cubren una extensión menor de $1 \%$ del área y albergan ocho especies del género Quercus, cuya distribución se restringe a los cañones y laderas bajas con condiciones favorables de humedad (Encina, 1996; Anónimo, 1998). Los bosques en cuestión han sido usados de manera tradicional por la población local, principalmente para obtener leña y madera, apacentamiento, aprovechamiento de plantas y acuíferos, extracción de tierra, además de actividades recreativas (Meganck et al., 1981; Anónimo, 1998). Dicha utilización ha ocasionado incendios frecuentes, sobreapacentamiento, erosión y cambios en el uso del suelo (Meganck et al., 1981; Portes, 1996). Amplias áreas de la cubierta forestal se encuentran con diversos grados de disturbio y se ha provocado su fragmentación (Anónimo, 1998). El presente estudio se realizó con el objetivo de conocer la estructura y composición florística de los bosques de encino de la zona sujeta a conservación ecológica Sierra de Zapalinamé. Se pretende que la información generada sirva de herramienta para la toma de decisiones en las actividades de fomento de esta vegetación y de su conservación.

\section{MATERIALES Y MÉTODOS}

Descripción del área de estudio

La información sobre la localización y geografía física del área de estudio fue tomada de Anónimo (1983) y Anónimo (1998); de acuerdo con estas publicaciones, la Sierra de Zapalinamé se localiza en el sureste de Coahuila, es una estribación de la Provincia Fisiográfica de la Sierra Madre Oriental, en el noreste de México, en la zona de transición entre esta provincia y el Desierto Chihuahuense. La sierra forma parte de los municipios de Saltillo y Arteaga y queda muy cerca de la ciudad de Saltillo. Se ubica entre los $25^{\circ} 15^{\prime} 00^{\prime \prime}-25^{\circ} 25^{\prime} 58.35^{\prime \prime}$ de latitud norte y los $100^{\circ} 47^{\prime} 14.5^{\prime \prime}-101^{\circ} 05^{\prime} 3.8^{\prime \prime}$ de longitud oeste (Fig. 1). Al norte y este colinda con la carretera 57 (México - Piedras Negras), al oeste con la carretera 54 (Saltillo - Zacatecas) y al sur está limitada por la coordenada de latitud $25^{\circ} 15^{\prime}$. La región pertenece a la Subprovincia de la Gran Sierra Plegada; el macizo incluye valles, planicies y montañas. La orientación de los pliegues transversales es de este a oeste, las altitudes van desde $1590 \mathrm{~m}$ en el pie de monte, hasta los $2200 \mathrm{~m}$ en los valles intermontanos, alcanzando su mayor elevación en el Cerro El Penitente a $3140 \mathrm{~m}$. La zona serrana generalmente se encuentra esculpida por cañones, con pendientes 


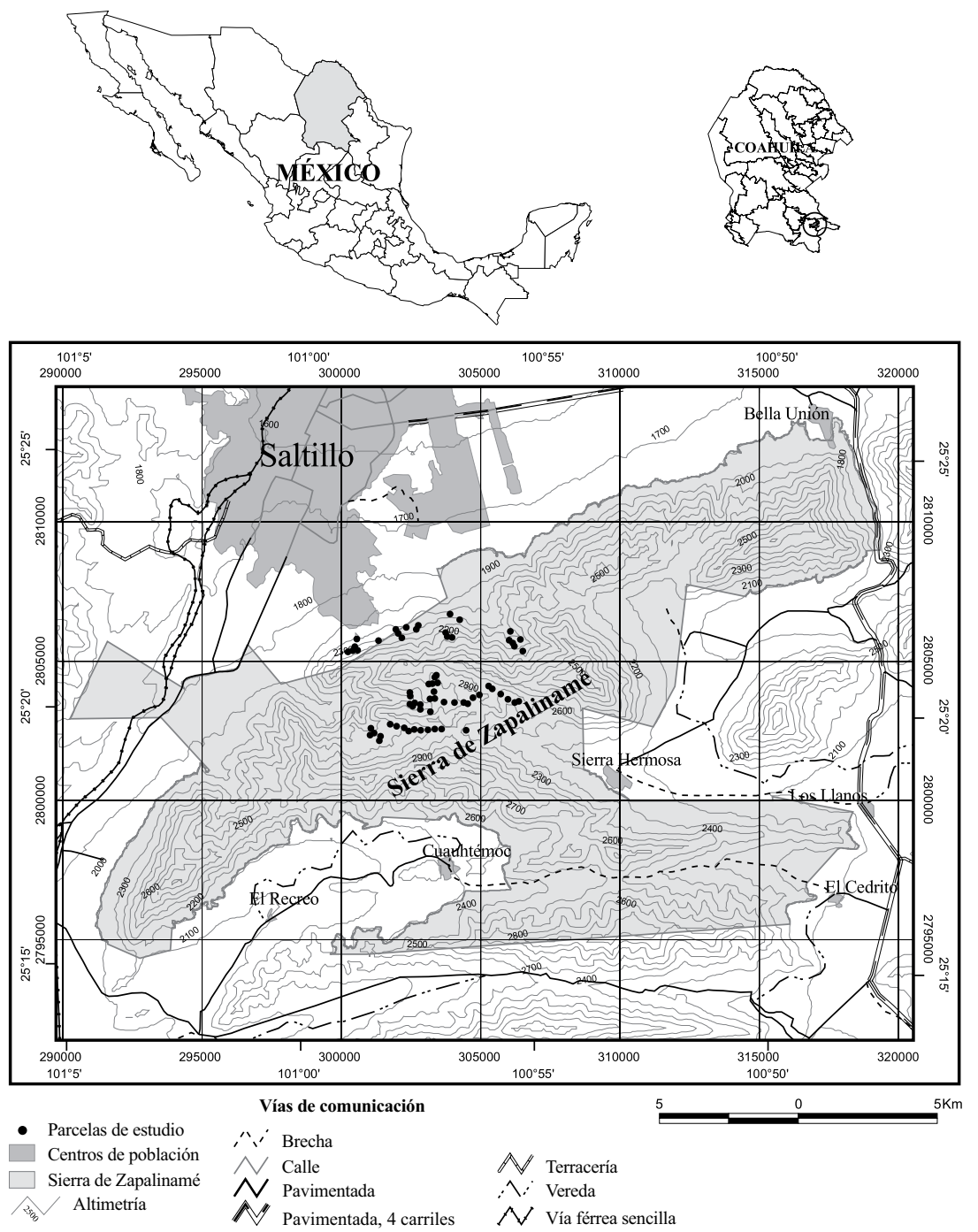

Fig. 1. Ubicación del área de estudio: Sierra de Zapalinamé.

abruptas y topografía accidentada. Las rocas que afloran en el área son sedimentarias marinas del Jurásico y Cretácico; las calizas cubren $43 \%$ del área, las areniscas y los conglomerados $17 \%$.

Los suelos aluviales ocupan casi $30 \%$ del área, son de profundidad variable y constituyen planicies con abanicos al pie del macizo, en los valles son profundos y 
con buen drenaje. Abundan los suelos litosoles y rendzinas, prevaleciendo ambos en casi $80 \%$ de la superficie del área. Los litosoles son superficiales y sobreyacen a la roca o caliche cementado, cubren $49 \%$ del área; los del tipo rendzina son pedregosos y someros, con una capa superficial de humus, sobre roca caliza o material rico en cal en el pie de monte y valles, representan $29 \%$. En menor proporción se localizan los xerosoles cálcico y feozem calcárico. El clima predominante en la región es seco BSkw, en las partes altas el clima es templado $\mathrm{C}\left(\mathrm{w}_{0}\right)$ (Anónimo, 1998). La caseta meteorológica más cercana está ubicada en Buenavista, Saltillo, Coah. $\left(23^{\circ} 38^{\prime} \mathrm{N}\right.$, $103^{\circ} 38^{\prime} \mathrm{W}$, a $1588 \mathrm{~m}$ s.n.m.), donde se registra una temperatura media anual de $16.9^{\circ}$ C y la precipitación media anual de $498 \mathrm{~mm}$, valores que se consideran próximos a los correspondientes de muchos parajes de la Sierra de Zapalinamé; las lluvias son de tipo convectivo, coincidiendo con los meses calientes del año.

Arce y Marroquín (1985) realizaron un estudio detallado de los tipos de vegetación de una parte del macizo montañoso y describieron 11 fitocenosis. En general la cubierta vegetal de las áreas con exposición sur está representada por matorrales rosetófilos y micrófilos. En las partes altas está integrada por bosque de pino y oyamel, en los cañones se localizan bosques de encino y en las laderas bajas de exposición norte y oeste se presenta el matorral submontano de rosáceas (Marroquín, 1976). Las formaciones mejor representadas son el bosque de pino que ocupa $14.09 \%$ de la superficie total del área protegida, el bosque de piñonero $12.54 \%$ y el bosque de piñonero con matorral xerófilo $9.55 \%$. En los cañones con más favorables condiciones de humedad se desarrollan bosques de encino (Arce y Marroquín, 1985); se trata de comunidades vegetales de porte bajo o mediano, con alturas entre 5 y $10 \mathrm{~m}$, de distribución irregular, con mayor frecuencia desarrollándose en altitudes entre 2200 y $2400 \mathrm{~m}$. Predominan en exposiciones norte y oeste, cuyas pendientes son mayores o iguales a $30 \%$, con tendencia a desarrollarse sobre suelos someros y rocosos (Ramírez, 1998; Arce y Marroquín, 1985).

Diseño y sitios de muestreo

Se identificaron dos localidades con bosques de encino en la Sierra de Zapalinamé: 1) Cañón de San Lorenzo, que incluye el cañón de Sierra Hermosa y Los Aguajes y 2) El Frente, que abarca los cañones cercanos a Lomas de Lourdes y Camino del Cuatro (Anónimo, 1998). Para el estudio de su vegetación se aplicó un diseño estratificado aleatorio. Se escogieron 66 sitios, equivalentes a 3.3 ha del encinar, su establecimiento en campo se realizó mediante el procedimiento de la compensación por pendiente propuesto por Medina (1983) y se ubicaron a una equidistancia de $200 \mathrm{~m}$, en un gradiente altitudinal a través de los cañones. Para realizar 
la medición de la vegetación se utilizó el método con parcela (Mueller-Dombois y Ellenberg, 1974). Las parcelas fueron circulares con un área de $500 \mathrm{~m}^{2}$, en las cuales se cuantificó la densidad y diámetro del tronco a $1.30 \mathrm{~m}$ del arbolado adulto (individuos con diámetro a $1.30 \mathrm{~m}$ de altura $>5 \mathrm{~cm}$ ), además de la cobertura y densidad de las plantas arbustivas. Los árboles juveniles (con diámetro a $1.30 \mathrm{~m}$ de altura $\leq 5$ $\mathrm{cm}$ y de $>1.30 \mathrm{~m}$ de altura), se midieron en un sitio de $50 \mathrm{~m}^{2}$ concéntrico a la parcela de $500 \mathrm{~m}^{2}$. Para evaluar la densidad de renuevos (plántulas y rebrotes $=$ individuos $\leq 1.30 \mathrm{~m}$ de altura) se establecieron cinco parcelas cuadradas de $1 \mathrm{~m}^{2}$, una al centro y cuatro en los puntos cardinales del círculo de $500 \mathrm{~m}^{2}$ (Olvera et al., 1996; Figueroa y Olvera, 2000; Galindo et al., 2002). Se recolectaron muestras botánicas que posteriormente se herborizaron, identificaron e incluyeron en el herbario ANSM (Universidad Autónoma Agraria Antonio Narro). Se registró el porcentaje de apertura del dosel, altitud (tomada de un altímetro-barómetro), pendiente (mediante una pistola Haga), posición topográfica (con respecto a un plano horizontal de referencia), exposición topográfica (tomada con una brújula) y profundidad del suelo (utilizando una barrena de $1.5 \mathrm{~m}$ ).

\section{Cálculos derivados de la medición de la vegetación}

Se calculó la densidad, cobertura o área basal y frecuencia de las especies dentro de las asociaciones del bosque de encino. Con la suma de las variables anteriores se calculó el índice de dominancia relativa por especie (Mueller-Dombois y Ellenberg, 1974). La estructura horizontal cuantitativa del estrato arbóreo se analizó en términos del área basal y densidad en las categorías mayores de $5 \mathrm{~cm}$ de diámetro a $1.30 \mathrm{~m}$. La descripción del estrato arbustivo consideró la cobertura y la densidad de las especies más abundantes.

\section{Análisis estadístico}

Para determinar las asociaciones vegetales en los bosque de encino, se utilizó el análisis de conglomerados a través del método de Ward (varianza mínima) (1963), mediante la técnica de Clasificación Jerárquica Politética Aglomerativa (Manly, 1986; Digby y Kempton, 1987; Estrada 1998) y el programa estadístico SAS versión 6.04 (Anónimo, 1985). Para obtener la clasificación de la vegetación se utilizó el índice de similitud de Motyka (Mueller-Dombois y Ellenberg, 1974), definido como $\mathrm{M}=2 \mathrm{c} /(\mathrm{a}+\mathrm{b}) \times 100$, en donde $\mathrm{c}=$ valor más bajo de densidad de las especies en común en dos sitios diferentes, a = valor de densidad total de las especies en el sitio $\mathrm{A}, \mathrm{b}=$ valor de densidad total de las especies en el sitio $\mathrm{B}$. Con los datos de densidad relativa de 68 diferentes plantas leñosas, se preparó una matriz 
de similitud-disimilitud para los 66 sitios. La matriz de disimilitud de distancias sirvió como base para efectuar el análisis de conglomerados (Cluster Analysis).

Asociaciones interespecíficas de las especies arbóreas

Se evaluó el grado de asociación entre parejas de especies arbóreas que contaran con al menos 10 individuos en un sitio. Con los valores de presencia y ausencia de las parejas seleccionadas se construyeron tablas de contingencia (Zar, 1984), cuya significancia fue evaluada por una prueba estadística de $X^{2}$ corregida de Yates (Ludwing y Reynolds, 1988), a través del programa Statistica. Asimismo la intensidad y el tipo de asociación (positiva o negativa) se evaluaron por medio de la técnica de correlación no paramétrica: Coeficiente de Correlación de Intervalos de Spearman (Ludwing y Reynolds, 1988). Este coeficiente varía de 0 (no asociada) a 1 (completamente asociada); el signo indica si la asociación es positiva (si el par de especies aparece con mayor frecuencia que la esperada por azar) o negativa (si ocurren juntas con menor frecuencia que la esperada) (Ludwing y Reynolds, 1988).

\section{RESULTADOS}

\section{Composición florística}

La flora vascular estimada de los bosques de encino de la Sierra de Zapalinamé está integrada por 259 especies y taxa infraespecíficos en 178 géneros y 67 familias, todos enumerados en el Apéndice. Las familias con mayor número de taxa son: Asteraceae (61 especies), Poaceae (27), Fabaceae (16) y Rosaceae (12). Los géneros mejor representados son Quercus (11 especies), Ageratina (7), Cheilanthes (5), Senecio (5), Salvia (5) y Stevia (5).

La fracción leñosa está compuesta por 68 especies, de las cuales 20 son árboles y 48 arbustos. De este total, 16\% está integrado por representantes del género Quercus (11), le siguen en orden decreciente cuatro gimnospermas de los géneros Pinus, Pseudotsuga, Cupressus y Juniperus, lo que representa 5.8\%, además de cinco miembros del género Ageratina con 7.3\%. El componente arbóreo está constituido por ocho especies de Quercus, los más frecuentes son: Q. saltillensis, Q. greggii y Q. mexicana, seguidos por Arbutus xalapensis y Juniperus flaccida. En el estrato arbustivo predominan Garrya glaberrima, Ageratina ligustrina, A. saltillensis, Salvia regla, Paxistima myrsinites y Stevia berlandieri, así como algunos elementos xerófilos, como Opuntia robusta, Yucca carnerosana y Agave spp. El estrato herbáceo está integrado por 189 especies, lo cual representa $72.9 \%$ de la flora vascular; 
las más comunes son: Artemisia ludoviciana, Pleopeltis guttata, Cheilanthes tomentosa, Achillea millefolium, Bromus carinatus, Piptochaetium fimbriatum, Salvia glechomifolia y Galium uncinulatum; entre las más escasas se encuentran las orquídeas Govenia liliacea y Goodyera oblongifolia. Se encontraron dos hemiparásitas: Phoradendron lanceolatum y P. tomentosum y dos epífitas: Tillandsia recurvata y $T$. erubescens. El número de elementos arbóreos y arbustivos permanece más o menos constante, mientras que la riqueza de plantas herbáceas, disminuye de forma notoria con el aumento de la altitud (Fig. 2).

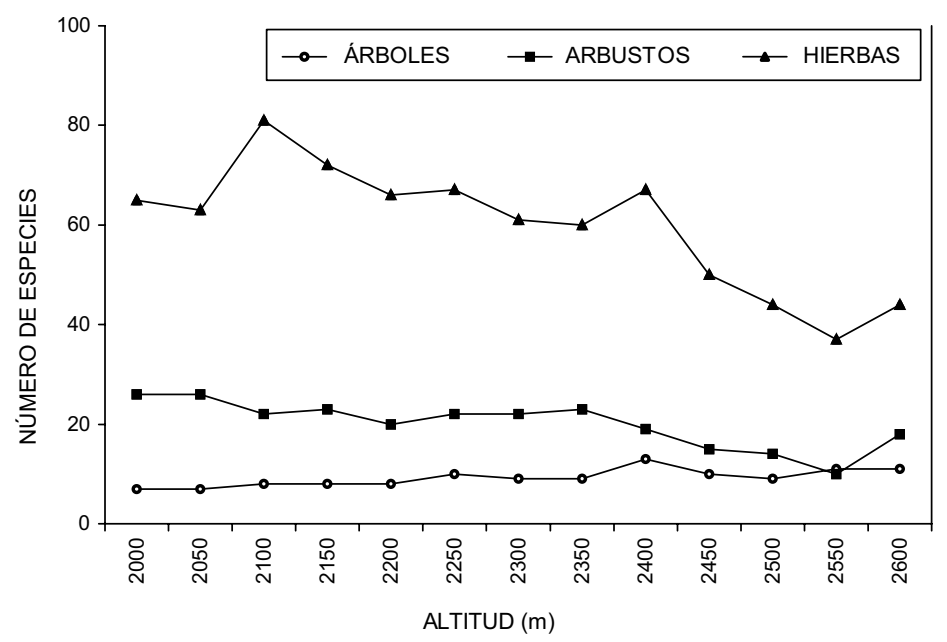

Fig. 2. Número de especies pertenecientes a las tres principales formas biológicas presentes a diferentes altitudes en los bosques de encino de la Sierra de Zapalinamé.

Asociación entre especies arbóreas del bosque de encino de la Sierra de Zapalinamé De las 37 parejas de especies arbóreas analizadas, la prueba de $\mathrm{X}^{2}$ arrojó significancia para 23, de las cuales con el coeficiente de intervalos de Spearman 10 mostraron asociación negativa, 13 parejas presentaron asociación positiva y 14 no tuvieron ninguna relación numérica significativa $(\mathrm{P}>0.05)$ (Cuadro 1). El valor de correlación más alto fue de 0.72, el cual se registró entre Quercus greggii y Q. mexicana. Quercus greggii es la especie más abundante en la zona y también se asoció positivamente con Pinus greggii, Arbutus xalapensis y Cupressus arizonica; mientras que con Quercus saltillensis, Q. grisea, Q. laeta y Juniperus flaccida presentó asociación negativa. Quercus saltillensis solamente mostró asociación positiva y significativa con $Q$. grisea, pero tuvo valores negativos con $Q$. greggii y $Q$. mexicana. Q. laeta 
Cuadro 1. Análisis de asociación entre especies arbóreas presentes en el bosque de encino de la Sierra de Zapalinamé.

\begin{tabular}{|c|c|c|c|c|}
\hline \multicolumn{2}{|c|}{ Especies analizadas } & \multirow{2}{*}{$\begin{array}{c}\mathrm{X}^{2} \text { Yates } \\
31.89 \\
\end{array}$} & \multirow{2}{*}{$\begin{array}{c}\mathrm{P}^{*} \\
0.000 \\
\end{array}$} & \multirow{2}{*}{$\begin{array}{c}\text { Coeficiente de } \\
\text { Spearman }\end{array}$} \\
\hline Quercus greggii & Quercus mexicana & & & \\
\hline & Quercus rugosa & 2.88 & $0.089 \mathrm{~ns}$ & 0.260 \\
\hline & Quercus saltillensis & 5.83 & 0.015 & -0.334 \\
\hline & Quercus sideroxyla & 0.52 & $0.467 \mathrm{~ns}$ & 0.164 \\
\hline & Quercus grisea & 21.28 & 0.000 & -0.601 \\
\hline \multirow[t]{3}{*}{ Quercus mexicana } & Quercus saltillensis & 5.86 & 0.015 & -0.334 \\
\hline & Quercus laeta & 23.90 & 0.000 & -0.633 \\
\hline & Quercus rugosa & 1.61 & $0.204 \mathrm{~ns}$ & -0.205 \\
\hline \multirow{3}{*}{$\begin{array}{l}\text { Quercus } \\
\text { saltillensis }\end{array}$} & Quercus laeta & 2.82 & $0.092 \mathrm{~ns}$ & 0.244 \\
\hline & Quercus grisea & 7.86 & 0.005 & 0.383 \\
\hline & Quercus laceyi & 2.10 & $0.146 \mathrm{~ns}$ & 0.229 \\
\hline \multirow[t]{4}{*}{ Quercus laeta } & Quercus laceyi & 18.78 & 0.000 & 0.577 \\
\hline & Quercus greggii & 42.47 & 0.000 & -0.835 \\
\hline & Quercus sideroxyla & 0.45 & $0.498 \mathrm{~ns}$ & -0.159 \\
\hline & Quercus grisea & 10.21 & 0.001 & 0.427 \\
\hline \multirow[t]{3}{*}{ Quercus grisea } & Quercus laceyi & 5.31 & 0.021 & 0.328 \\
\hline & Quercus mexicana & 12.92 & 0.000 & -0.474 \\
\hline & Quercus rugosa & 2.41 & $0.120 \mathrm{~ns}$ & -0.243 \\
\hline \multirow[t]{5}{*}{ Pinus greggii } & Quercus greggii & 19.15 & 0.000 & 0.571 \\
\hline & Quercus mexicana & 13.19 & 0.000 & 0.478 \\
\hline & Quercus saltillensis & 1.56 & $0.211 \mathrm{~ns}$ & -0.191 \\
\hline & Quercus laeta & 17.83 & 0.000 & -0.552 \\
\hline & Quercus sideroxyla & 2.99 & $0.083 \mathrm{~ns}$ & 0.288 \\
\hline \multirow[t]{6}{*}{ Arbutus xalapensis } & Quercus greggii & 11.62 & 0.000 & 0.460 \\
\hline & Quercus laeta & 17.90 & 0.000 & -0.562 \\
\hline & Quercus mexicana & 11.29 & 0.000 & 0.453 \\
\hline & Pinus greggii & 6.57 & 0.010 & 0.356 \\
\hline & Quercus saltillensis & 2.87 & $0.089 \mathrm{~ns}$ & -0.255 \\
\hline & Juniperus flaccida & 0.91 & $0.338 \mathrm{~ns}$ & -0.157 \\
\hline
\end{tabular}


Cuadro 1. Continuación.

\begin{tabular}{|l|l|l|l|c|}
\hline \multirow{4}{*}{ Juniperus flaccida } & Quercus saltillensis & 3.10 & $0.078 \mathrm{~ns}$ & 0.253 \\
\cline { 2 - 5 } & Quercus laeta & 4.27 & 0.038 & 0.286 \\
\cline { 2 - 5 } & Quercus greggii & 5.30 & 0.021 & -0.314 \\
\cline { 2 - 5 } & Quercus mexicana & 6.15 & 0.013 & -0.335 \\
\cline { 2 - 5 } & Quercus grisea & 4.34 & $0.067 \mathrm{~ns}$ & 0.257 \\
\hline \multirow{4}{*}{$\begin{array}{l}\text { Cupressus } \\
\text { arizonica }\end{array}$} & Quercus greggii & 4.27 & 0.038 & 0.300 \\
\cline { 2 - 5 } & Quercus mexicana & 3.14 & 0.076 & 0.262 \\
\cline { 2 - 5 } & Quercus laeta & 1.51 & $0.218 \mathrm{~ns}$ & -0.197 \\
\hline
\end{tabular}

*1 grado de libertad $\mathrm{X}^{2} \mathrm{t}=3.84, \mathrm{p}<0.05,95 \%$ confiabilidad.

$\mathrm{X}^{2} \mathrm{t}=6.63, \mathrm{p}<0.01,99 \%$ confiabilidad.

$\mathrm{X}^{2} \mathrm{t}=10.82, \mathrm{p}<0.001,99.9 \%$ confiabilidad.

ns $=$ no significativa.

presentó liga significativa y positiva con $Q$. laceyi y $Q$. grisea y Juniperus flaccida. $Q$. grisea también la tuvo significativa y positiva con $Q$. laeta y $Q$. laceyi. Arbutus xalapensis y Juniperus flaccida son muy frecuentes en los bosques de encino estudiados, pero la asociación entre ambas especies fue negativa y no significativa. La primera "prefiere" establecerse en los bosques de clima templado subhúmedo, donde cohabita con Quercus greggii (0.46), Q. mexicana (0.45) y Pinus greggii (0.35), mientras que la segunda se presenta con mayor frecuencia en los templado-semisecos, asociándose de manera significativa con Q. laeta $(0.28)$.

Clasificación de los bosques de encino estudiados

La agrupación jerárquica de los sitios se muestra en el dendrograma (Fig. 3). Se identificaron dos grandes grupos ( 1 y 2 ) diferenciados por un valor de $\mathrm{r}^{2}=$ 72.83. De esta forma los bosques de Quercus greggii - Q. mexicana representan el grupo 1, al que corresponden 40 localidades, mientras que los bosques de Quercus saltillensis - Q. laeta forman el grupo 2, el cual comprende 26. Los índices de similitud de Motyka son de 32.30\% (grupo 1) y 40.36\% (grupo 2) respectivamente. Estos conjuntos están definidos por la dominancia de una o dos especies, más que por una combinación particular de varias; la nomenclatura con la que se denominó a cada agrupación estuvo determinada por los elementos más abundantes. En los dos grupos identificados se reconocen a su vez dos subdivisiones, que se definen a continuación. 


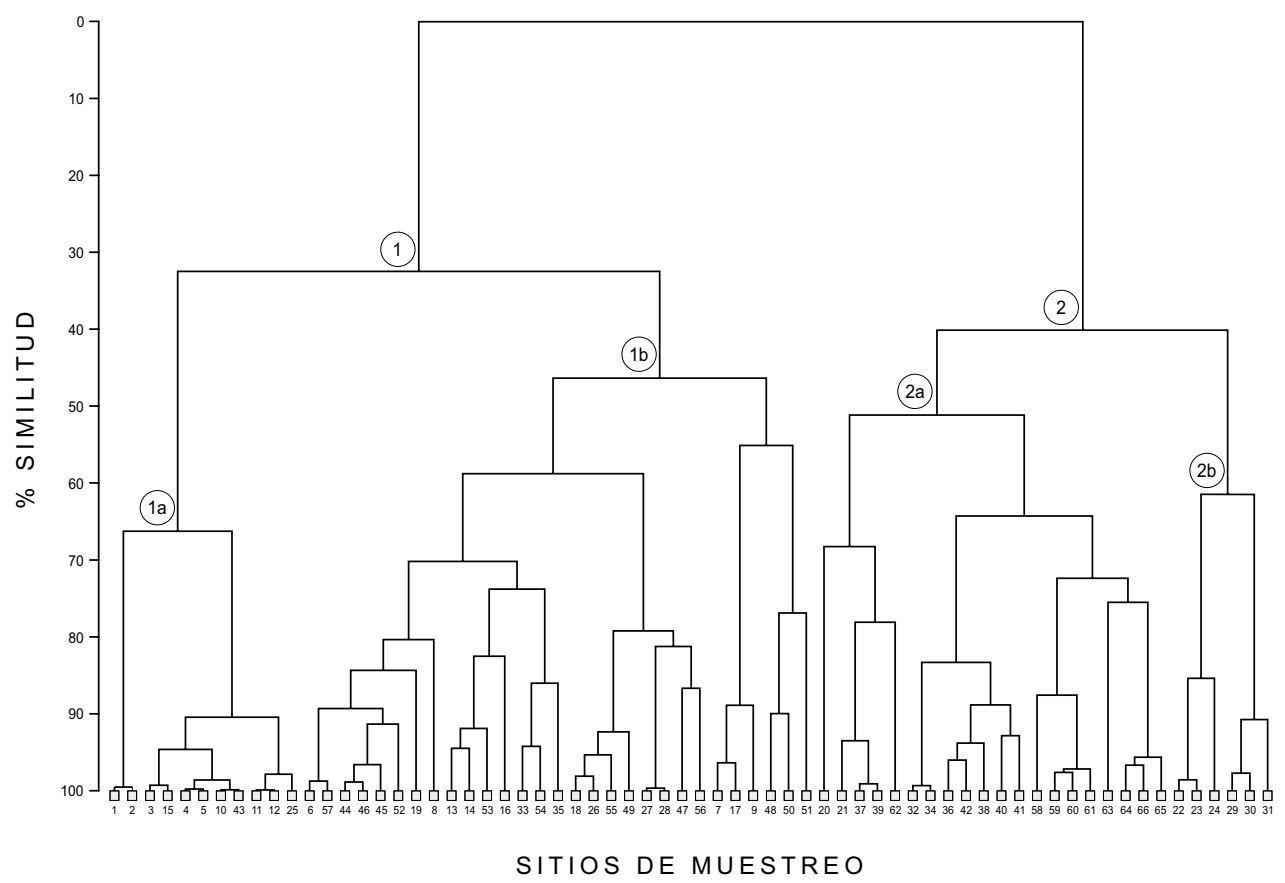

Fig. 3. Agrupación de los sitios de muestreo ubicados en los bosques de encino de la Sierra de Zapalinamé en función de la similitud florística de su estrato arbóreo.

Grupo 1. Bosque de Quercus greggii - Q. mexicana

Subgrupo 1a. Es una agrupación registrada en 11 sitios de muestreo, con un índice de similitud de Motyka de 66.29\%. Se localiza en altitudes de 2380 a 2600 m. Quercus greggii es el componente más abundante y dominante fisonómico, con $85 \%$ de densidad relativa. Q. mexicana sólo alcanza $5 \%$ de la misma, pero es frecuente a través de los inventarios. Q. sideroxyla tiene una distribución restringida y es abundante en localidades cercanas a elevaciones de 2600 m s.n.m. Pinus greggii, Quercus saltillensis y Arbutus xalapensis cuentan con individuos aislados a través de este bosque y registran menos de 3\% de participación.

Subgrupo 1b. Esta categoría corresponde a casi la mitad de los sitios de muestreo, con un índice de similitud de $46.37 \%$. Se presenta en altitudes de 2280 a 2600 m. Quercus greggii ocupa 56\% de la densidad del estrato arbóreo, seguida de Q. mexicana con $14 \%$ y Q. saltillensis $8 \%$. Arbutus xalapensis, Pinus greggii y Juniperus flaccida son frecuentes, pero con muy baja densidad. Quercus rugo- 
sa y $Q$. grisea ocupan cada una $5.5 \%$ de la densidad de los árboles y tienen una distribución restringida; la primera de ellas crece en cañones protegidos, mientras que la segunda prospera en bosques mas secos. Otras especies más escasas y con distribución restringida son Cupressus arizonica, Pseudotsuga menziesii y Fraxinus cuspidata.

Grupo 2. Bosque de Quercus saltillensis - Q. laeta

Subgrupo 2a. Variante inventariada en 30\% de los sitios de muestreo, conformados con un índice de similitud de $51.16 \%$. Se desarrolla en altitudes de 2000 a $2300 \mathrm{~m}$. La especie dominante es $Q$. laeta, con valor de $45 \%$ de densidad, seguida por Q. saltillensis con 35\%. Juniperus flaccida, Q. grisea y Arbutus xalapensis son medianamente frecuentes. Sin embargo, $Q$. grisea es la más abundante de estas tres, con $5 \%$ de la densidad. Se presenta asimismo $Q$. laceyi, pero es menos asidua. Q. greggii y $Q$. mexicana tienen una distribución restringida y son escasas.

Subgrupo 2b. Comunidad censada en seis sitios de muestreo, los que se encuentran integrados con un coeficiente de similitud de $61.44 \%$. Se localiza en una altitud media de $2330 \mathrm{~m}$. Está dominada por Quercus grisea y Q. saltillensis, que ocupan $55 \%$ y $28 \%$, respectivamente, de la densidad del estrato arbóreo. Juniperus flaccida y Arbutus xalapensis son frecuentes pero con baja densidad, mientras que Q. greggii y Q. laeta ocupan $8.9 \%$, y solamente en un sitio son más abundantes que las especies anteriores.

A continuación se describen los dos principales grupos identificados para los bosques de encino de la Sierra de Zapalinamé. Se incluyen aspectos de su composición florística, así como características fisonómicas y aspectos estructurales (densidad, diámetro medio, área basal, cobertura y valor de importancia) de los estratos arbustivo y arbóreo.

\section{Grupo 1. Bosque de Quercus greggii - Q. mexicana}

Se localiza en el cañón de San Lorenzo. En esta comunidad se registraron 153 especies vegetales (18 arbóreas). Quercus greggii y Q. mexicana dominan fisonómicamente con alturas de 8 a $10 \mathrm{~m}$, y alcanzan los mayores valores de importancia (Cuadro 2) y del coeficiente de Spearman, siendo este último de 0.72 (Cuadro 1). En pocos lugares $Q$. sideroxyla forma bosques escasos de encino-pino a $2500 \mathrm{~m}$ de altitud asociándose con Quercus greggii y Pinus greggii. Quercus rugosa aparece en algunas cañadas con mayor humedad y convive con mayor frecuencia con $Q$. greggii; Q. saltillensis es escaso. De manera aislada se establece Pinus greggii; su porte 
es de 14-16 m, por lo que sobresale de la altura media del encinar y como parte de la composición arbórea se presentan árboles bajos de Arbutus xalapensis. El estrato arbustivo está integrado por 37 especies; las de valores de importancia más altos son Garrya glaberrima, Ageratina saltillensis y A. ligustrina, (Cuadro 2). Se registraron además dos epifitas (Tillandsia recurvata y T. erubescens) y dos hemiparásitas (Phoradendron tomentosum y P. lanceolatum). El estrato herbáceo lo forman 103 especies. Las más frecuentes son: Pleopeltis guttata, Achillea millefolium, Artemisia ludoviciana, Cheilanthes tomentosa, Bromus anomalus y Malaxis brachystachya. La planta más abundante es el helecho Pleopeltis guttata.

Densidad y área basal. El estrato arbóreo (elementos adultos y juveniles) presenta una densidad total de 2277 árboles ha ${ }^{-1}$ (Cuadro 2). El número de individuos de tronco más grueso es drásticamente menor en comparación con los correspondientes a otras categorías diamétricas (Fig. 4). Estas últimas varían desde 5 hasta $60 \mathrm{~cm}$ (Fig. 4), la mayor cantidad de árboles se registra en la de $10 \mathrm{~cm}$, seguida por la correspondiente a la clase de $5 \mathrm{~cm}$, ambas están dominadas por individuos de Quercus greggii. Quercus mexicana es relativamente importante entre 15 y $30 \mathrm{~cm}$; la clase de menor densidad ( 0.5 árboles ha $\left.{ }^{-1}\right)$ es la de $60 \mathrm{~cm}$ y está constituida solamente por Quercus rugosa; Pinus greggii forma las categorías de 50 y $55 \mathrm{~cm}$ con 1.5 árboles $\mathrm{ha}^{-1}$ Las especies con la mayor contribución en área basal corresponden a Quercus greggii y Q. mexicana (Cuadro 2).

La cantidad de renuevos observada es de 196 individuos ha-1 (Fig. 5). Quercus greggii contribuye con $66 \%$, seguida de $Q$. mexicana, $Q$. sideroxyla y $Q$. saltillensis con 22,18 y 11 individuos ha-1 ${ }^{-1}$ espectivamente. Se registra una altura de rebrotes de 0.30 a $1.20 \mathrm{~m}$. Para el caso de las plantas juveniles la densidad es de 95 individuos $\mathrm{ha}^{-1}$, de los cuales $Q$. greggii tiene 57, lo que representa $61 \%$ del total, seguida por Q. saltillensis con 11 árboles $\mathrm{ha}^{-1}(12 \%)$. El estrato arbustivo mostró mayor riqueza de especies que el arbóreo y estuvo dominado por Garrya glaberrima (Cuadro 2), seguido por Ageratina saltillensis y A. ligustrina.

Grupo 2. Bosques de Quercus saltillensis - Q. laeta

Esta comunidad se ubica en altitudes de 2000 a $2300 \mathrm{~m}$ en los cañones de El Frente, que corresponde a la exposición norte del macizo montañoso y en la porción sur del cañón de San Lorenzo. Ocupa una superficie menor en comparación con la del bosque de Quercus greggii - Q. mexicana. Está integrada por 202 especies de las cuales 13 son árboles. Quercus saltillensis, Q. laeta y Q. grisea son las que registran los valores de importancia más altos (Cuadro 3). Los coeficientes de Spearman entre estos taxones son menores de 0.45 , sin embargo, la asociación entre Quercus laeta y 
Cuadro 2. Atributos estructurales promedio calculados de 39 sitios para las principales especies leñosas del bosque de Quercus greggii - Q. mexicana. Valor de importancia = Densidad relativa + Área basal o cobertura relativa + Frecuencia relativa / 3.

\begin{tabular}{|c|c|c|c|c|c|}
\hline \multicolumn{6}{|c|}{ ÁRBOLES } \\
\hline Especie & $\begin{array}{l}\text { Densidad } \\
\left(\text { ind } / \mathrm{ha}^{-1}\right)\end{array}$ & $\begin{array}{l}\text { Diámetro medio } \\
\text { del tronco }(\mathrm{cm})\end{array}$ & \multicolumn{2}{|c|}{$\begin{array}{l}\text { Área basal } \\
\left(\mathrm{m}^{2} / \mathrm{ha}^{-1}\right)\end{array}$} & $\begin{array}{c}\text { Valor de } \\
\text { importancia }\end{array}$ \\
\hline Quercus greggii & $1480 \pm 49.33$ & $7.7 \pm 3.22$ & \multicolumn{2}{|c|}{$9.00 \pm 0.295$} & 41.27 \\
\hline Quercus mexicana & $284 \pm 10.89$ & $13.3 \pm 7.30$ & \multicolumn{2}{|c|}{$5.68 \pm 0.250$} & 18.16 \\
\hline Pinus greggii & $40 \pm 2.35$ & $28.4 \pm 14.99$ & \multicolumn{2}{|c|}{$3.50 \pm 0.207$} & 9.60 \\
\hline Quercus saltillensis & $122 \pm 9.25$ & $7.5 \pm 2.85$ & \multicolumn{2}{|c|}{$0.75 \pm 0.062$} & 6.37 \\
\hline Arbutus xalapensis & $47 \pm 2.35$ & $8.1 \pm 3.57$ & \multicolumn{2}{|c|}{$0.30 \pm 0.018$} & 5.55 \\
\hline Quercus rugosa & $98 \pm 14.20$ & $10.7 \pm 5.95$ & \multicolumn{2}{|c|}{$1.08 \pm 0.160$} & 4.16 \\
\hline Quercus grisea & $87 \pm 17.22$ & $8.2 \pm 3.40$ & \multicolumn{2}{|c|}{$0.55 \pm 0.105$} & 2.57 \\
\hline Juniperus flaccida & $18 \pm 1.95$ & $7.9 \pm 4.63$ & \multicolumn{2}{|c|}{$0.10 \pm 0.015$} & 2.47 \\
\hline Cupressus arizonica & $15 \pm 1.82$ & $13.6 \pm 10.34$ & \multicolumn{2}{|c|}{$0.33 \pm 0.057$} & 2.14 \\
\hline Quercus sideroxyla & $48 \pm 10.17$ & $8.8 \pm 5.49$ & \multicolumn{2}{|c|}{$0.57 \pm 0.117$} & 2.02 \\
\hline Otras especies (8) & $38 \pm 6.45$ & $9.0 \pm 5.91$ & \multicolumn{2}{|c|}{$6.97 \pm 0.263$} & 5.69 \\
\hline \multicolumn{6}{|c|}{ ARBUSTOS } \\
\hline Especie & Densidad (ind $/ \mathrm{ha}^{-1}$ ) & \multicolumn{2}{|c|}{ Cobertura relativa $(\%)$} & \multicolumn{2}{|c|}{ Valor de importancia } \\
\hline Garrya glaberrima & 726 & \multicolumn{2}{|c|}{44.80} & \multicolumn{2}{|c|}{25.14} \\
\hline Agertina saltillensis & 384 & \multicolumn{2}{|l|}{7.53} & \multicolumn{2}{|r|}{9.19} \\
\hline Ageratina ligustrina & 365 & \multicolumn{2}{|l|}{7.52} & \multicolumn{2}{|r|}{8.84} \\
\hline Paxistima myrsinites & 527 & \multicolumn{2}{|l|}{3.52} & \multicolumn{2}{|r|}{7.80} \\
\hline Arbutus xalapensis & 150 & \multicolumn{2}{|l|}{7.33} & \multicolumn{2}{|r|}{7.03} \\
\hline Stevia berlandieri & 160 & \multicolumn{2}{|l|}{4.02} & \multicolumn{2}{|r|}{5.53} \\
\hline Otras especies (31) & 995 & 25.28 & & & 36.47 \\
\hline
\end{tabular}

Q. saltillensis no es significativa. Son frecuentes individuos bajos de Juniperus flaccida y Arbutus xalapensis, sin embargo, crecen de manera aislada. En los cañones ubicados en altitudes mayores se localizan poblaciones poco abundantes de Quercus greggii, mientras que en las menores se encuentran árboles bajos y escasos de Quercus laceyi. El estrato arbustivo está integrado por 46 especies, de las que Ageratina saltillensis, Salvia regla y Stevia berlandieri resultan ser las dominantes. El herbáceo está compuesto por 146 elementos; siendo Artemisia ludoviciana, Cologania pallida, Cheilanthes alabamensis, Piptochaetium fimbriatum y Pellaea atropurpurea las plan- 
Encina Domínguez et al.: Composición y aspectos estructurales de los bosques de encino de Zapalinamé

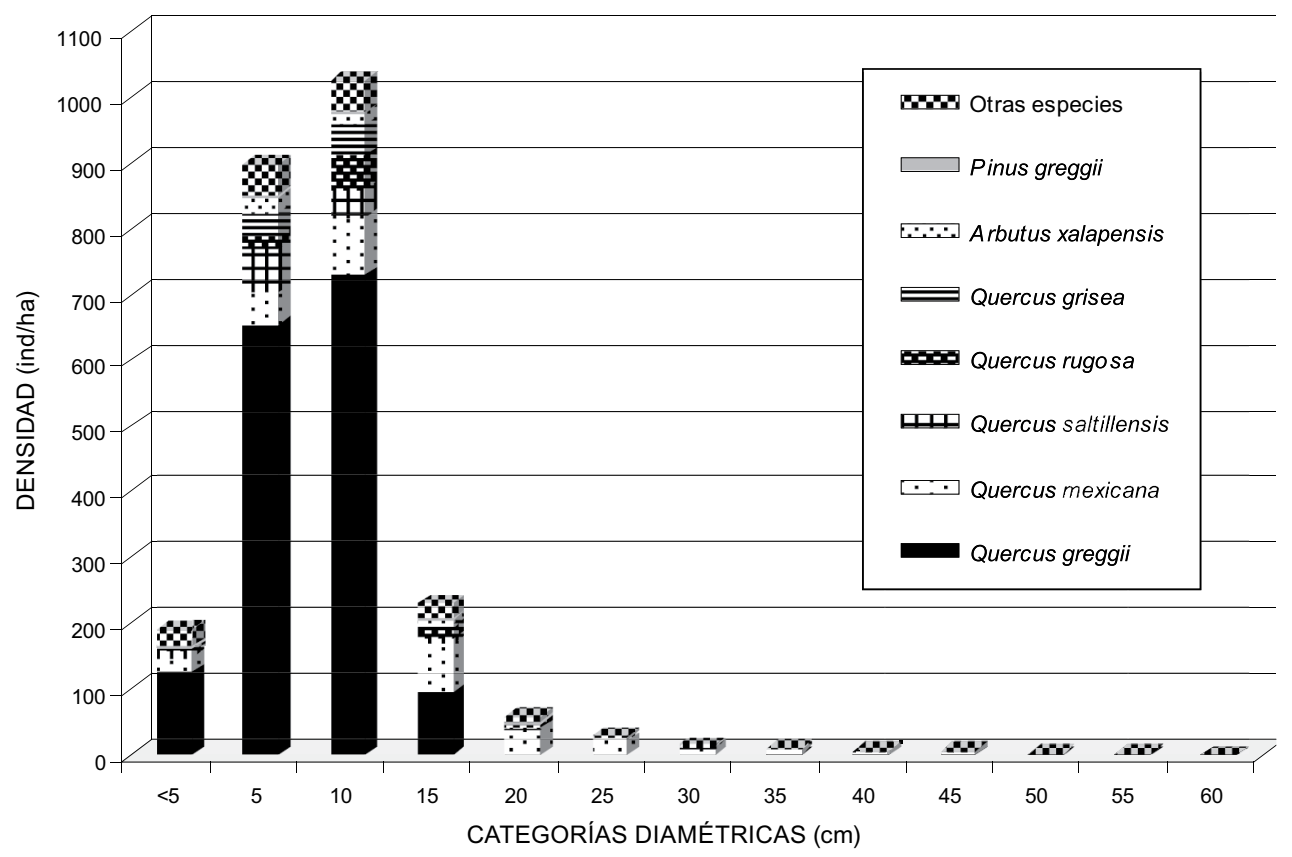

Fig. 4. Distribución promedio del arbolado por categorías diamétricas en el bosque de Quercus greggii - Q. mexicana. La categoría $<5 \mathrm{~cm}$ abarca de $0-5 \mathrm{~cm}$; la de $5 \mathrm{~cm}$ abarca de 5 a $9.99 \mathrm{~cm}$; la de $10 \mathrm{~cm}$ abarca de 10 a 14.99 y así sucesivamente.

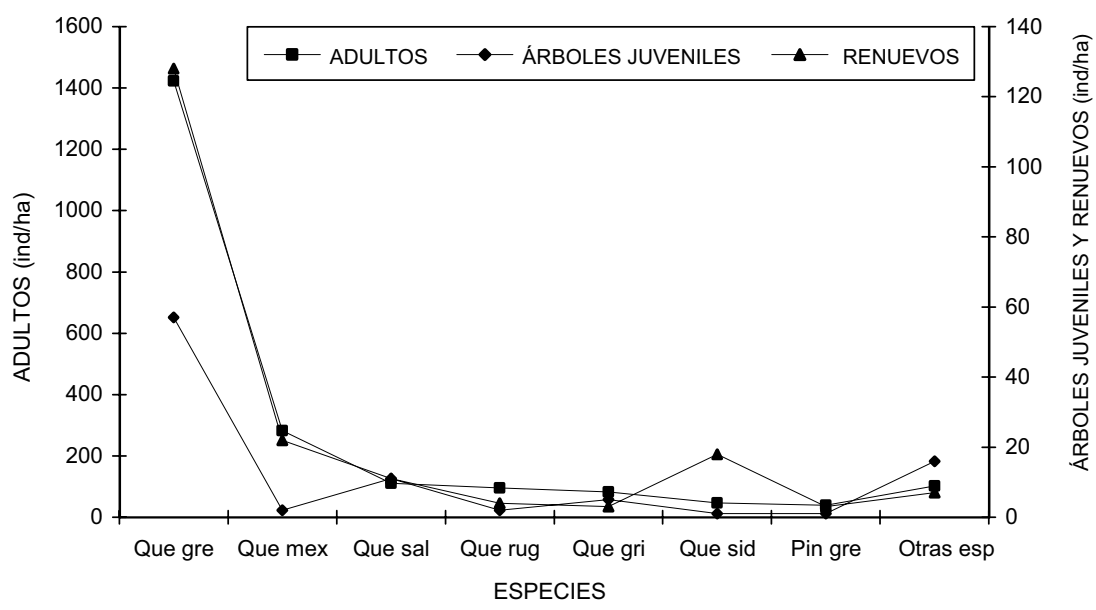

Fig. 5. Densidad de especies arbóreas por clase de edad para los bosques de Quercus greggii - Q. mexicana. 
tas más frecuentes; sin embargo, Astragalus greggii, Smallanthus uvedalius y Heliopsis parvifolia son más abundantes.

Densidad y área basal. El estrato arbóreo (adultos y juveniles) registra una densidad total de 2346 árboles ha-1 (Cuadro 3). La densidad promedio se encuentra distribuida desde la categoría de 5 hasta la de $30 \mathrm{~cm}$, el mayor número de componentes ha ${ }^{-1}$ se observa en la de $10 \mathrm{~cm}$, seguido por la de $5 \mathrm{~cm}$. Las categorías diamétricas entre 5-10 y $15-20 \mathrm{~cm}$, están dominadas por individuos de Quercus saltillensis y $Q$. laeta, Quercus grisea lo está en más escasa proporción, mientras que en la de 25-30 $\mathrm{cm}$ se tiene la menor densidad (2 árboles ha-1), donde Quercus saltillensis es el único elemento (Fig. 6). Al igual que para el bosque de Quercus greggii - Q. mexicana, el número de árboles de mayores categorías diamétricas es drásticamente más pequeño. Las especies con la mayor contribución en área basal corresponden a Quercus saltillensis y Q. laeta (Cuadro 3).

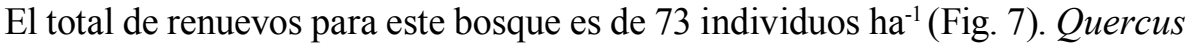
grisea representa $33 \%$, seguida de Q. laeta y Q. greggii con $25 \%$ y $19 \%$. La densidad de las plantas juveniles es de 80 individuos ha-1. De las especies que ahí crecen, $Q$. saltillensis cuenta con 21 plantas ha ${ }^{-1}$ que representan $27 \%$, seguida por Q. laeta con 19, es decir, (23\%). El estrato arbustivo está dominado por Ageratina saltillensis, con los más altos valores de densidad y cobertura, seguida por Salvia regla y Stevia berlandieri las cuales poseen 449 y 269 individuos ha ${ }^{-1}$ respectivamente (Cuadro 3).

\section{DISCUSIÓN}

Composición y asociación de especies de los bosques de encino

La flora de los bosques de encino estudiados constituye $8.35 \%$ del total de las plantas vasculares citadas para Coahuila por Villarreal (2001). De las 409 especies y taxa infraespecíficos registrados por Arce (1980) para la Sierra de Zapalinamé, los 259 elementos encontrados en los bosques de encino representan $63 \%$ de la flora. La diversidad arbórea del bosque de encino estudiado es baja, si se compara con la de otras comunidades similares de México (Rzedowski, 1978; Challenger, 1998). La dominancia fisonómica está definida por dos o más especies al igual que en otros encinares de nuestro país (Rzedowski, 1978). De la flora de los de la Sierra de Zapalinamé, 72\% está integrada por elementos herbáceos y, a semejanza de otros bosques mexicanos de Quercus, las familias de plantas vasculares mejor representadas son Asteraceae, Poaceae y Fabaceae (Rzedowski, 1978). La riqueza florística es más alta en elevaciones bajas, donde existe mayor influencia de incendios, así como 
Cuadro 3. Atributos estructurales promedio calculados de 27 sitios para las principales especies leñosas del bosque de Quercus saltillensis - Q. laeta. Valor de importancia = Densidad relativa + Área basal o cobertura relativa + Frecuencia relativa / 3.

\begin{tabular}{|c|c|c|c|c|c|c|}
\hline \multicolumn{7}{|c|}{ ÁRBOLES } \\
\hline Especie & $\begin{array}{l}\text { Densidad } \\
\left(\mathrm{ind} / \mathrm{ha}^{-1}\right)\end{array}$ & & $\begin{array}{l}\text { iámetro medio } \\
\text { lel tronco }(\mathrm{cm})\end{array}$ & \multicolumn{2}{|c|}{$\begin{array}{c}\text { Área basal } \\
\left(\mathrm{m}^{2} / \mathrm{ha}^{-1}\right)\end{array}$} & $\begin{array}{c}\text { Valor de } \\
\text { importancia }\end{array}$ \\
\hline Quercus saltillensis & $876 \pm 28.75$ & & $9.08 \pm 3.50$ & \multicolumn{2}{|c|}{$6.62 \pm 0.22$} & 32.23 \\
\hline Quercus laeta & $741 \pm 33.54$ & & $9.35 \pm 3.37$ & \multicolumn{2}{|c|}{$5.93 \pm 0.28$} & 29.92 \\
\hline Quercus grisea & $368 \pm 28.60$ & & $7.37 \pm 2.89$ & \multicolumn{2}{|c|}{$1.87 \pm 0.17$} & 13.65 \\
\hline Juniperus flaccida & $72 \pm 4.76$ & & $9.57 \pm 5.54$ & \multicolumn{2}{|c|}{$0.73 \pm 0.04$} & 7.85 \\
\hline Arbutus xalapensis & $33 \pm 2.40$ & & $8.98 \pm 3.34$ & \multicolumn{2}{|c|}{$0.26 \pm 0.02$} & 5.02 \\
\hline Quercus greggii & $132 \pm 22.98$ & & $6.81 \pm 2.60$ & \multicolumn{2}{|c|}{$0.66 \pm 0.11$} & 3.96 \\
\hline Quercus laceyi & $83 \pm 9.65$ & & $6.41 \pm 2.34$ & \multicolumn{2}{|c|}{$0.33 \pm 0.04$} & 3.72 \\
\hline Otras especies (6) & $41 \pm 3.22$ & & $9.9 \pm 4.48$ & \multicolumn{2}{|c|}{$0.95 \pm 0.10$} & 3.65 \\
\hline \multicolumn{7}{|c|}{ ARBUSTOS } \\
\hline Especie & \multicolumn{2}{|c|}{ Densidad (ind $/$ ha $^{-1}$ ) } & \multicolumn{2}{|c|}{ Cobertura relativa $(\%)$} & \multicolumn{2}{|c|}{ Valor de importancia } \\
\hline $\begin{array}{l}\text { Ageratina } \\
\text { saltillensis }\end{array}$ & \multicolumn{2}{|c|}{456} & \multicolumn{2}{|c|}{15.17} & \multicolumn{2}{|c|}{12.02} \\
\hline Salvia regla & \multicolumn{2}{|l|}{449} & \multicolumn{2}{|l|}{15.54} & \multicolumn{2}{|r|}{11.32} \\
\hline Stevia berlandieri & \multicolumn{2}{|l|}{269} & \multicolumn{2}{|l|}{6.68} & \multicolumn{2}{|r|}{7.10} \\
\hline Garrya ovata & \multicolumn{2}{|l|}{231} & \multicolumn{2}{|l|}{7.43} & \multicolumn{2}{|r|}{6.32} \\
\hline Ageratina ligustrina & \multicolumn{2}{|l|}{202} & \multicolumn{2}{|l|}{7.01} & \multicolumn{2}{|r|}{5.88} \\
\hline Agave gentryi & \multicolumn{2}{|l|}{102} & \multicolumn{2}{|l|}{7.35} & \multicolumn{2}{|r|}{5.45} \\
\hline Bouvardia ternifolia & \multicolumn{2}{|l|}{235} & \multicolumn{2}{|l|}{2.61} & \multicolumn{2}{|r|}{5.12} \\
\hline Otras especies (39) & \multicolumn{2}{|l|}{1363} & 38.21 & & & 46.79 \\
\hline
\end{tabular}

a causa de la cercanía del matorral submontano de rosáceas, el cual domina en áreas adyacentes al bosque (Arce y Marroquín, 1985). El estrato arbóreo de los encinares de la Sierra de Zapalinamé muestra afinidad florística con el de los de Nuevo León y del centro de México, ya que comparten encinos como Quercus laeta, Q. mexicana, Q. rugosa, Q. laceyi y Q. greggii (Valdés y Aguilar, 1983; Müller-Using, 1994; Espinosa, 2001), que extienden su distribución hasta el sureste de Coahuila a través de la Sierra Madre Oriental (Encina, 1996). Además, comparte Quercus grisea, Q. rugosa, Q. laeta y Q. sideroxyla con los bosques de Durango, México (GonzálezElizondo et al. 1993). 


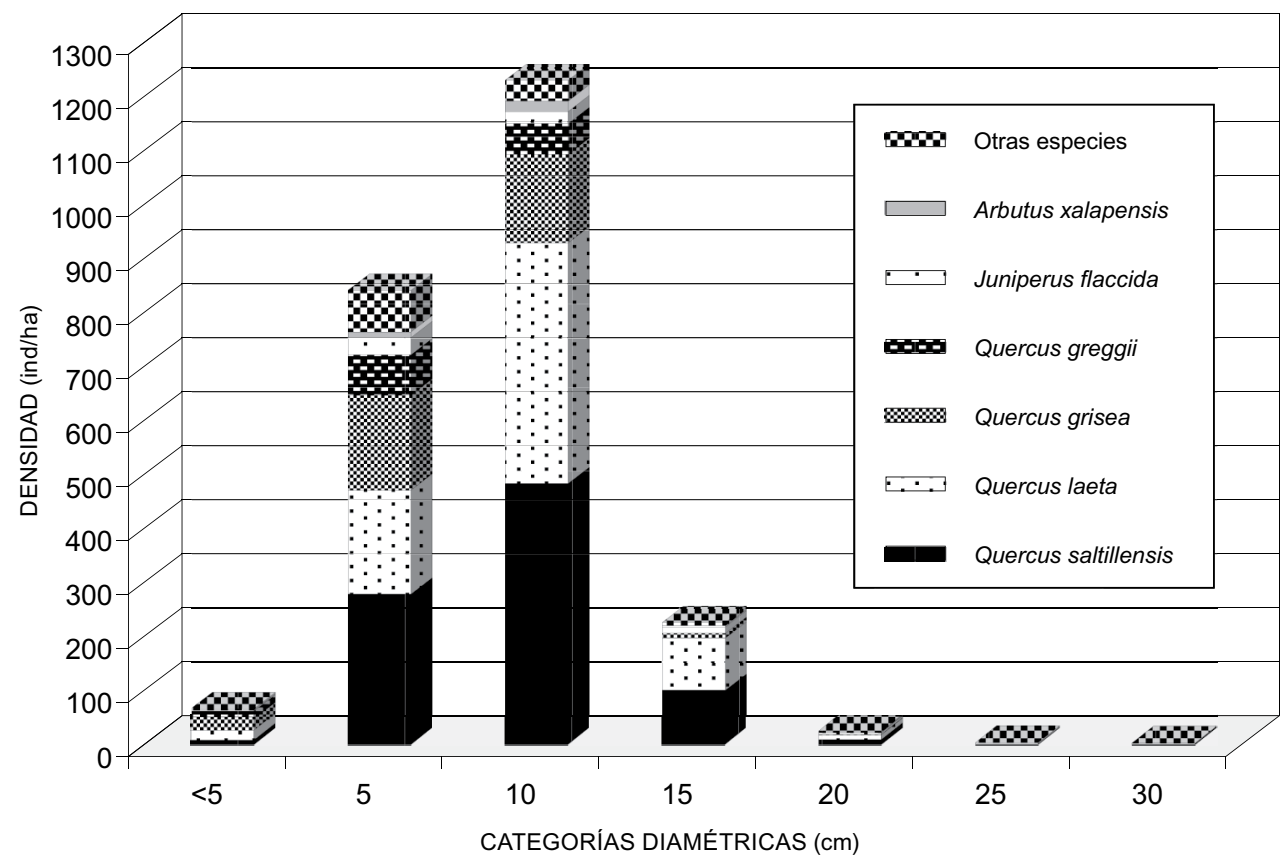

Fig. 6. Distribución promedio del arbolado por categorías diamétricas en el bosque de Quercus saltillensis - Q. laeta. La categoría $<5 \mathrm{~cm}$ abarca de $0-5 \mathrm{~cm}$; la de $5 \mathrm{~cm}$ abarca de 5 a $9.99 \mathrm{~cm}$; la de $10 \mathrm{~cm}$ abarca de 10 a 14.99 y así sucesivamente.

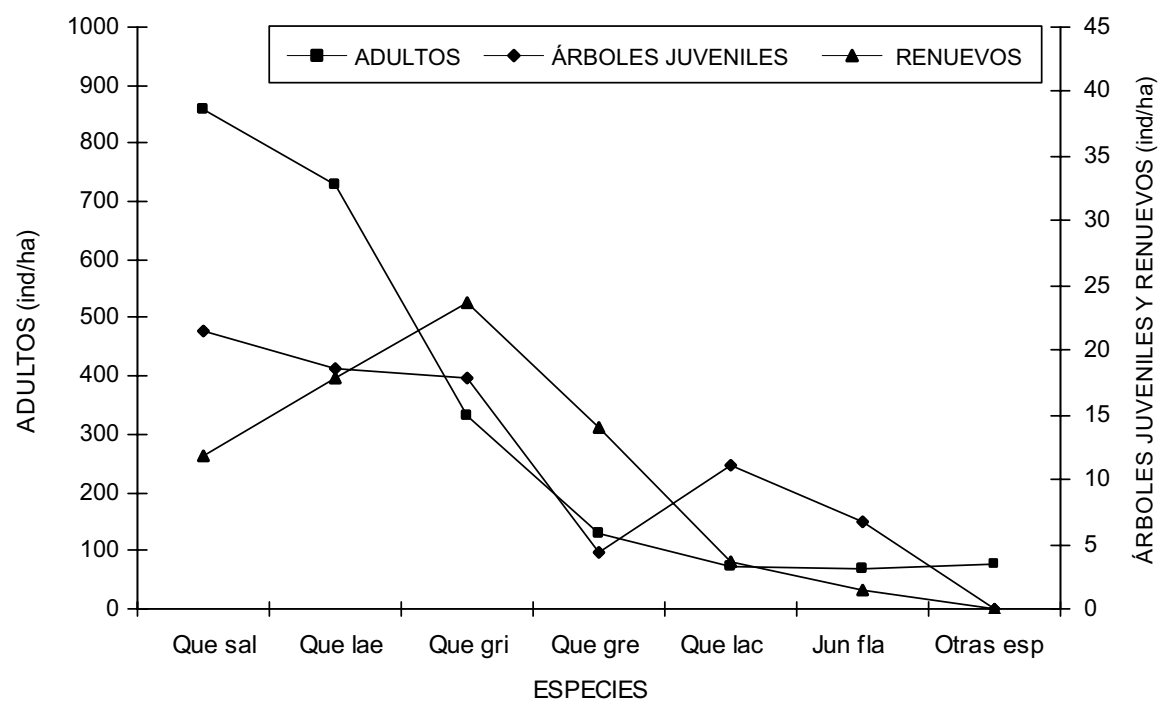

Fig. 7. Densidad de especies arbóreas por clases de edad para los bosques de Quercus saltillensis - Q. laeta. 
En general, el número de especies herbáceas y, en menor proporción, de arbustos disminuye significativamente con el aumento de la altitud, mientras que el de árboles aumenta ligeramente. Vásquez y Givnish (1998) obtuvieron resultados similares para la Sierra de Manantlán, Jalisco. Dichos autores consideran que tal patrón se debe al hecho de que los bosques que se desarrollan en altitudes menores son más deciduos, exhiben mayor apertura del dosel y por ello resultan más secos; al respecto Ramírez et al. (2001) señalan que la apertura del dosel en los bosques determina la composición de especies arbustivas y herbáceas, y favorece la colonización de matorrales xerófilos. Los disturbios más acentuados en estas comunidades propician el establecimiento de una elevada riqueza de plantas herbáceas. Lo anterior es aplicable a los bosques de Quercus saltillensis - Q. laeta, que son más abiertos y permiten una mayor cantidad de radiación solar a nivel de los estratos inferiores, lo que contribuye a una gran diversidad y abundancia de especies anuales. En cambio, los encinares situados en altitudes superiores, como son los de Quercus greggii - Q. mexicana, se caracterizan por ser más densos, más perennifolios y estar conformados por un número algo mayor de elementos arbóreos, lo que se debe a que prosperan en ambientes más húmedos y con menor influencia de la aridez.

Estructura de los bosques de encino de la Sierra de Zapalinamé

La estructura de los bosques de encino de la zona de estudio es sencilla y la densidad, así como el área basal de su arbolado son bajas, en comparación con las de la mayoría de los encinares mexicanos (Rzedowski, 1978; González-Elizondo et al., 1993; Saldaña y Jardel, 1991; González et al., 1995a; Zavala, 1995) o de los bosques mesófilos dominados por especies de Quercus del centro y sur de México, donde existe una mayor riqueza arbórea (Puig et al., 1987; Santiago y Jardel, 1993; Ruíz et al., 2000). Los de la Sierra de Zapalinamé presentan semejanzas estructurales con los encinares que se desarrollan en los macizos montañosos del centro y norte de Coahuila, como por ejemplo la Sierra de la Paila (Villarreal, 1994), Sierra del Carmen (Wood et al., 1999), así como la Sierra de San Carlos, Tamaulipas (Briones, 1991) y la Sierra Madre Oriental en Nuevo León (Valdés y Aguilar, 1983; MüllerUsing, 1994). En general, los diámetros, área basal y altura de los bosques de esos lugares son similares, pero existen diferencias florísticas notables.

En cuanto a las comunidades de este estudio, se registraron pocas diferencias entre la densidad de los bosques de Quercus greggii - Q. mexicana y los de Quercus saltillensis - Q. laeta, aunque sus áreas basales resultaron ser contrastantes. El estrato arbóreo en el de Quercus greggii - Q. mexicana está integrado 
por 18 especies pero es dominado sólo por la primera, mientras que el de Quercus saltillensis - Q. laeta incluye 13 elementos, de las que prevalecen tres. Quercus greggii aporta $65 \%$ de la densidad total en la comunidad donde se presenta, aunque no desarrolla troncos muy gruesos y solamente ocupa $31 \%$ del valor de área basal total. En general la magnitud de esta última está influenciada por una mayor densidad de individuos con diámetros de $10 \mathrm{~cm}$, aun cuando el número de árboles sea elevado. Los valores de importancia de Quercus greggii, $Q$. saltillensis y $Q$. laeta son resultado de una alta densidad y frecuencia de individuos con diámetros delgados y área basal baja. En contraste, los de Q. mexicana y Pinus greggii derivan de un área basal alta debido a la presencia de individuos con diámetros gruesos pero con baja densidad. Pinus greggii es una conífera frecuente en los bosques de Quercus greggii - Q. mexicana, cuyos troncos más corpulentos determinan su posición entre las especies con mayor valor de importancia superando a Quercus saltillensis, Q. rugosa y $Q$. grisea.

La forma de la distribución de las categorías diamétricas de las comunidades investigadas es característica de bosques incoetáneos o de edades no uniformes constituidos por árboles de troncos de grosor diverso (Daniel et al., 1982; Smith, 1986). Tal estructura señala la existencia de una mayor cantidad de diámetros de tamaño pequeño que de talla grande e indica una escasa regeneración. Semejante distribución es propia también de especies tolerantes a la sombra en bosques húmedos (Murphy y Farrar, 1988; Martínez-Ramos y Álvarez-Buylla, 1995). La densidad total de renuevos en los dos encinares estudiados es 269 individuos ha ${ }^{-1}$ y 175 plantas juveniles ha ${ }^{-1}$, es baja en comparación con los valores registrados para otras áreas de México. En los bosques subtropicales de la Sierra de Manantlán, Jalisco (Saldaña y Jardel, 1991) se obtuvo una lectura media de regeneración de 197 individuos ha ${ }^{-1}$. Eckelmann (1995) para bosques de encino - pino de Nuevo León registró $1200 \mathrm{ha}^{-1}$, cantidad que aún contrasta con una regeneración de 8000 a 20000 individuos ha-1 encontrada para la misma comunidad vegetal de los Altos de Chiapas (Galindo et al., 2002). En los encinares de la Sierra de Zapalinamé la densidad de plantas juveniles y renuevos es menor que la de los árboles adultos, lo cual puede ser debido a la competencia de arbustos, efecto de pastoreo y la baja producción de bellotas, factores que impiden el establecimiento de estos encinos.

En los bosques de encino de la Sierra de Zapalinamé con mayor humedad y doseles cerrados, el estrato arbustivo es abundante y dominan especies de los géneros Garrya, Ageratina, Paxistima y Arbutus, calificadas de afinidad esciófila. Dada su profusión, estas especies impiden el establecimiento de árboles juveniles y 
la regeneración de los bosques; con la apertura del dosel tales plantas son sustituidas por arbustos xéricos, propios de los matorrales rosetófilo y submontano de rosáceas (Arce y Marroquín, 1985). En los encinares de la Sierra de Zapalinamé, en especial en el de Quercus saltillensis - Q. laeta, la condición de mayor aridez podría explicar la presencia de arbustos, pues estos son resistentes al fuego y se adaptan a las áreas impactadas. Así, la elevada cobertura y dominancia de plantas arbustivas sobre los individuos juveniles y renuevos de los árboles sugiere que el encinar podría quedar reemplazado en el mediano plazo por matorrales. Una secuencia similar ha sido documentada por Casas et al. (1995) para algunos bosques de encino de Durango, México.

El disturbio antropogénico y su impacto en la estructura y composición florística

En la Sierra de Zapalinamé los bosques de Quercus han sufrido modificaciones tanto en superficie como en distribución, debido a la presión antropogénica y a los incendios (Portes, 2001); en algunas áreas son de tipo secundario, mantenidos así por el constante disturbio. La permanencia de la mayoría de los encinos ha sido parcialmente favorecida por su alta tolerancia al fuego (Abrams, 1992), debida a su corteza gruesa y resistente, además de la capacidad para generar rebrotes a partir de tocones, raíces y tallos maduros (Spurr y Barnes, 1982; Zavala, 2000). Sin embargo, estas características no garantizan su regeneración, ya que después de un incendio se propician condiciones microclimáticas y de disponibilidad de nutrientes (Hobbs y Huenneke, 1992) que favorecen la invasión y el establecimiento de arbustos, plantas herbáceas anuales y zacates (Ramírez et al., 2001). El efecto del fuego en la composición florística y estructura varía dependiendo de su intensidad y frecuencia (Rzedowski, 1978; Attiwill, 1994). En los bosques de Zapalinamé que han sufrido incendios superficiales se ha favorecido la presencia de un estrato herbáceo denso dominado por Smallanthus uvedalius, Heliopsis parvifolia y Cologania pallida, además de Astragalus greggii (obs. personal). Se sabe que los incendios estimulan los rebrotes de algunos encinos; sin embargo, otras especies de Quercus no dependen de tales retoños para su regeneración, por lo que el fuego puede ser perjudicial para sus plántulas (Zavala, 2000). Debido al incremento en la disponibilidad de forraje en las áreas incendiadas del bosque de encino, pronto se efectúa el apacentamiento del ganado, el cual ocasiona el ramoneo y con ello la eliminación de renuevos de los árboles, impidiendo la recuperación del bosque.

Además, tal disturbio compacta y/o remueve el suelo, creando un hábitat propicio para el establecimiento de especies invasoras anuales de tipo ruderal, disper- 
sadas por el ganado (Hobbs y Huenneke, 1992, Pettit et al., 1995). En los muestreos realizados se detectaron algunas malezas de los géneros Solidago, Bidens, Taraxacum, Asphodelus, Oenothera y Physalis. Estas plantas actualmente no son comunes, sin embargo, de continuar el libre ramoneo del ganado y la expansión de la zona urbana, tales malezas podrán ser más abundantes e inclusive reemplazar a algunas especies nativas del bosque (Vetaas, 1997).

El apacentamiento extensivo de bovinos es común en los bosques de México (Challenger, 1998), sin embargo, su efecto ecológico ha sido escasamente estudiado (Hernández et al., 2000); se considera que el disturbio antropogénico afecta de manera negativa la densidad de renuevos (Ramírez, 2003). Nosotros observamos que en los bosques de Quercus greggii - Q. mexicana el pastoreo de reses es el principal agente de disturbio, mientras que en el bosque de $Q$. saltillensis - $Q$. laeta el de cabras es más importante. Los rebrotes de los encinos son ramoneados, sobre todo en las épocas de sequía, pero también sus plántulas mostraron huellas de ser mordidas; tales daños a la regeneración también han sido registrados por González-Elizondo et al. (1993) de Durango, México. De esta forma el sobreapacentamiento en los bosques en el largo plazo, podría ocasionar su desaparición y con ello la pérdida de la diversidad biológica (Fleischner, 1994; Hernández et al., 2000).

La baja densidad de renuevos en las comunidades estudiadas indica un pobre reclutamiento de los árboles dominantes. Esta condición prevalece en otros encinares de México (Saldaña y Jardel, 1991; González-Elizondo et al., 1993; Müller-Using, 1994; Casas et al., 1995), Norteamérica (Crow, 1988; Mensing, 1992) y Eurasia (Vetaas, 2000). La regeneración de los encinos proviene de semillas además de brotes a partir de rizomas (Zavala y García, 1997); en el área estudiada de estas especies se han observado mayormente rebrotes y pocas plántulas; situación similar se ha registrado en bosques de Nuevo León (Eckelmann, 1995; Fisher y López, 1995). Quercus greggii presenta más capacidad de la reproducción vegetativa, mientras que Q. mexicana se regenera sólo de semillas (Zavala y García, 1997). Las escasas plántulas de encino observadas en la región de estudio se encuentran en la comunidad de Quercus greggii - Q. mexicana, donde prevalece mayor humedad, no han ocurrido incendios y la presión del apacentamiento es menor; una situación similar ha sido señalada para bosques de encino por Vetaas (2000), quien menciona que la regeneración está ligada a sitios con condiciones ambientales favorables y que no están sujetos a disturbio.

Debido a que los bosques de Quercus saltillensis - Q. laeta se desarrollan en laderas más cercanas a la zona urbana, han sido más afectados por los incendios 
ocurridos en los años de 1994 y 1998 (Zárate, com. pers.) y por el apacentamiento de animales, factores que explicarían su mayor apertura del dosel y la reducción de su superficie. La asociación dominada por Quercus greggii y Q. mexicana es la más representativa del bosque de encino bien desarrollado, se ubica en el fondo del cañón de San Lorenzo, en donde anteriormente existían corrientes de agua permanentes, se desarrolla en elevaciones mayores y lugares menos accesibles, por lo cual tiene una mayor cobertura del dosel y muestra un mayor grado de conservación.

Probablemente como consecuencia del disturbio, los árboles en el área de estudio presentan una gran abundancia de hemiparásitas (muérdago). De igual manera que en otros encinares de México (Rzedowski, 1978), las especies más comunes de estas últimas pertenecen al género Phoradendron, las cuales crecen principalmente sobre Quercus mexicana y Q. grisea.

En los bosques húmedos de mayores latitudes en Norteamérica el reemplazo de encinos por otras dicotiledóneas arbóreas se debe a la eliminación (o supresión) de los incendios (Abrams y Downs, 1990; Abrams, 1992), mientras que en las montañas del sureste mexicano la fuerte presión antropogénica (extracción selectiva de árboles para leña y madera, deforestación con fines agropecuarios y apacentamiento) ha ocasionado que los encinares sean sustituidos por bosques de pino (González et al.,1991; González et al., 1995a; González y Ramírez, 1999). Además, allí los de Quercus se vuelven más xéricos, sufren modificaciones del sustrato y cambios en la estructura y composición de especies (González et al., 1995b; Ramírez et al., 2001; Galindo et al., 2002, Ramírez, 2003).

Para bosques de clima templado semiseco, como los de la Sierra de Zapalinamé, donde no existe competencia de árboles caducifolios tolerantes a la sombra, se considera que los encinos pueden mantener sus poblaciones estables y no depender del fuego para su regeneración (Abrams, 1992), pero por otro lado su regeneración se ve disminuida debido al apacentamiento extensivo (Mensing, 1992) y al efecto de los incendios. Algunos bosques de Quercus de México han desaparecido porque sus especies no resisten la acción del fuego y han sido sustituidos por matorrales xerófilos (Casas et al., 1995) o zacatales secundarios (Rzedowski, 1978). El cambio en el uso del suelo y la presión de las actividades humanas han ocasionado procesos de desertificación en la región de estudio (Portes, 2001). Esto ha propiciado una tendencia general de avance de los matorrales en sustitución de los bosques de encino (Arce y Marroquín, 1985). 


\section{AGRADECIMIENTOS}

El primer autor reconoce el apoyo del Consejo Nacional de Ciencia y Tecnología para realizar esta investigación, la cual forma parte de la tesis de maestría en ciencias. Agradecemos las facilidades brindadas en la realización del presente trabajo al herbario ANSM de la Universidad Autónoma Agraria Antonio Narro; a Jorge S. Marroquín, por sus sugerencias a la primera versión del manuscrito y a Efrén Mata Rocha por la revisión crítica del manuscrito final; a Erika M. Reyna, por la elaboración de la figura 1. Se dan las gracias al personal del área de protección Sierra de Zapalinamé por su apoyo durante el desarrollo del trabajo de campo. Los revisores anónimos aportaron valiosas sugerencias que incrementaron la calidad del manuscrito.

\section{LITERATURA CITADA}

Abrams, M. D. 1992. Fire and the development of oak forest. Bioscience 42: 346-353.

Abrams, M. D. y J. A. Downs. 1990. Successional replacement of old-growth white oak by mixed mesophytic hardwoods in southwest Pennsylvania. Can. J. For. Res. 20: 18641870.

Anónimo. 1983. Síntesis geográfica de Coahuila. México. Instituto Nacional de Estadística Geografía e Informática. Secretaría de Programación y Presupuesto. México, D.F. $163 \mathrm{pp}$.

Anónimo. 1985. SAS user's guide: statistics. 5.0 edition, SAS Institute, Cary, North Carolina, U.S.A. Inc. 56 pp.

Anónimo. 1996. Decreto de la Sierra de Zapalinamé como área natural protegida, con carácter de zona sujeta a conservación ecológica. Periódico Oficial del Gobierno de Coahuila. Tomo CIII. No. 83. Saltillo, Coah.

Anónimo. 1998. Programa de manejo de la zona sujeta a conservación ecológica "Sierra de Zapalinamé". Secretaría de Desarrollo Social, Gobierno del estado de Coahuila. Saltillo, Coah. 179 pp.

Arce, G. L. 1980. Adición al estudio de la vegetación y la flora del cañón de San Lorenzo, Saltillo, Coah., México. Tesis de licenciatura. Facultad de Ciencias Biológicas, Universidad Autónoma de Nuevo León. Monterrey, N.L. 92 pp.

Arce, G. L. y J. S. Marroquín. 1985. Las unidades fisonómico-florísticas del cañón de San Lorenzo, Saltillo, Coahuila, México. Biotica 10(4): 369-393.

Attiwill, P. M. 1994. The disturbance of forest ecosystems: the ecological basis for conservative management. For. Ecol. Manage. 63: 247-300.

Briones, V. O. 1991. Sobre la flora, vegetación y fitogeografía de la Sierra de San Carlos, Tamaulipas. Acta Bot. Mex. 16: 15-44. 
Casas, S. R., S. González-Elizondo. y J. A. Tena F. 1995. Estructura y tendencias sucesionales en vegetación de clima templado semi-seco en Durango, México. Madroño 42(4): 501-515.

Challenger, A. 1998. Utilización de los ecosistemas terrestres de México. Pasado, presente y futuro. Comisión Nacional para el Conocimiento y Uso de la Biodiversidad - Instituto de Biología - Universidad Nacional Autónoma de México - Agrupación Sierra Madre, S.C. México, D.F. 813 pp.

Crow, T. R. 1988. Reproductive mode and mechanisms for self-replacement of northern red oak (Quercus rubra) - a review. Forest Sci. 34: 19-40.

Daniel, T. W., J. A. Helmes y F. S. Baker. 1982. Principios de silvicultura. Mc Graw Hill. México, D.F. 490 pp.

Digby, P. G. y R. A. Kempton. 1987. Multivariate analysis of ecological communities. Chapman \& Hall. Londres. 206 pp.

Eckelmann, C. M. 1995. Regeneración y dinámica sucesional de un bosque de pino-encino en la Sierra Madre Oriental en el noreste de México. In: Marroquín, J. S. (ed.). Memorias del tercer seminario nacional sobre utilización de encinos. Universidad Autónoma de Nuevo León. Rep. Cient. No. Esp. 15(1): 199-212.

Encina, D. J. 1996. Distribución y aspectos ecológicos del género Quercus L. en el estado de Coahuila, México. Tesis de licenciatura. Universidad Autónoma Agraria Antonio Narro. Buenavista, Saltillo, Coah. 123 pp.

Espinosa, G. J. 2001. Fagaceae. In: Rzedowski, G. C. de, J. Rzedowski y colaboradores. Flora fanerogámica del Valle de México. 2a. ed. Instituto de Ecología, A.C. y Comisión Nacional para el Conocimiento y Uso de la Biodiversidad. Pátzcuaro, Mich. pp. 8191.

Estrada, C. E. 1998. Ecología del matorral submontano en el Estado de Nuevo León, México. Tesis doctoral. Facultad de Zootecnia, Universidad Autónoma de Chihuahua. Chihuahua, Chih. 190 pp.

Figueroa, B. L. y M. Olvera. 2000. Dinámica de la composición de especies en bosques de Quercus crassipes H. \& B. en Cerro Grande, Sierra de Manantlán, México. Agrociencia 34: 91-98.

Fisher, M. y R. López A. 1995. Observaciones sobre la regeneración de dos especies de encinos en Santa Rosa, Iturbide, N. L. In: Marroquín, J. S. (ed.). Memorias del tercer seminario nacional sobre utilización de encinos. Universidad Autónoma de Nuevo León. Rep. Cient. No. Esp. 15(1): 234-237.

Fleischner, T. L. 1994. Ecological cost of livestock grazing in western North America. Conserv. Biol. 8: 629-644.

Galindo, J. L., M. González E. y P. Quintana A. 2002. Tree composition and structure in disturbed stands with varying dominance by Pinus spp. in the highlands of Chiapas, México. Plant Ecol. 162(2): 259-272.

González, M., P. F. Quintana, N. Ramírez. y P. Gaytán. 1991. Secondary succession in disturbed Pinus-Quercus forests of the highlands of Chiapas, Mexico. J. Veg. Sci. 2: 351-360. 
González, M., N. Ramírez, P. F. Quintana y M. Martínez. 1995a. La utilización de encinos y la conservación de la biodiversidad en Los Altos de Chiapas. In: Marroquín, J. S. (ed.). Memorias del tercer seminario nacional sobre utilización de encinos. Universidad Autónoma de Nuevo León. Rep. Cient. No. Esp. 15(1): 183-197.

González, M., S. Ochoa, N. Ramírez y P. Quintana. 1995b. Current land-use trends and conservation of old-growth forest habitats in the highlands of Chiapas, Mexico. In: Wilson, M. y S. Sader (eds.). Conservation of neotropical migratory birds in Mexico. Maine Agric. For. Exper. Stn. Misc. Publ. 727: 190-198.

González, M. y N. Ramírez. 1999. El disturbio antrópico y la conservación y restauración de los bosques en los Altos de Chiapas, México. Simposio sobre Manejo, Conservación y Restauración de Recursos Naturales en México, Sociedad Botánica de México, A.C. Morelia, Mich. 15 pp.

González-Elizondo, S., M. González-Elizondo y A. Cortés. 1993. Vegetación de la reserva de la biosfera "La Michilía”, Durango, México. Acta Bot. Mex. 22: 1-104.

Hernández, V. G., L. R. Sánchez, T. F. Carmona, M. R. Pineda y R. Cuevas. 2000. Efecto de la ganadería extensiva sobre la regeneración arbórea de los bosques de la Sierra de Manantlán. Madera y Bosques 6(2): 13-28.

Hobbs, R. J. y L. F. Huenneke. 1992. Disturbance, diversity and invasion: implications for conservation. Conserv. Biol. 6: 324-337.

Jardel, P. E. 1986. Efecto de la explotación forestal en la estructura y regeneración del bosque de coníferas de la vertiente oriental del Cofre de Perote, Ver., México. Biotica 2(4): 247-270.

Jardel, P. E. 1991. Perturbaciones naturales y antropogénicas y su influencia en la dinámica sucesional de los bosques de Las Joyas, Sierra de Manantlán, Jalisco. Tiempos de Ciencia 22: 9-26.

Ludwing, J. A. y J. F. Reynolds. 1988. Statistical ecology: a primer on methods and computing. John Wiley and Sons. Nueva York. 337 pp.

Manly, B. F. 1986. Multivariate methods. Chapman \& Hall. Londres. 159 pp.

Marroquín, J. S. 1976. Vegetación y florística del nordeste de México. I. Aspectos sinecológicos en Coahuila. Rev. Soc. Mex. Hist. Nat. 36: 69-101.

Martínez-Ramos, M. y E. Álvarez-Buylla. 1995. Ecología de poblaciones de plantas en una selva húmeda de México. Bol. Soc. Bot. Méx. 56: 121-153.

Medina, B. R. 1983. Delimitación de sitios circulares de muestreo en investigación forestal. Ciencia Forestal 8(43): 3-25.

Meganck, R. A., J. López., F. Rodríguez C. y V. Serrato C. 1981. Plan de manejo para el uso múltiple del cañón de San Lorenzo. Universidad Autónoma Agraria Antonio Narro, Organización de los Estados Americanos. Saltillo, Coah. 129 pp.

Mensing, S. A. 1992. The impact of the European settlement on blue oak (Quercus douglasii) regeneration and recruitment in the Tehachapi Mountains, California. Madroño 39(1): 36-46.

Mueller-Dombois, D. y H. Ellenberg. 1974. Aims and methods of vegetation ecology. John Wiley \& Sons. Inc. Nueva York. 547 pp. 
Müller-Using, B. 1994. Contribuciones al conocimiento de los bosques de encino y pino-encino en el noreste de México. Reporte Científico No. Especial 14. Facultad de Ciencias Forestales, Universidad Autónoma de Nuevo León. Linares, Nuevo León. 181 pp.

Murphy, P. A. y R. M. Farrar. 1988. A framework for stand structure projection of unevenaged loblolly - short leaf pine stands. For. Sci. 34: 321-332.

Nixon, K. C. 1993. The genus Quercus in Mexico. In: Ramamoorthy, T. P., R. Bye, A. Lot y J. Fa (eds.). Biological diversity of Mexico: origins and distribution. Capítulo 16, pp. 447-458. Oxford University Press. Nueva York. 812 pp.

Olvera, M., S. Moreno y B. Figueroa. 1996. Sitios permanentes para la investigación silvícola. Manual para su establecimiento. Libros del Instituto Manantlán. Universidad de Guadalajara, Guadalajara, Jal. 55 pp.

Pettit, N. E., R. H. Froend y P. G. Ladd. 1995. Grazing in remnant woodland vegetation: changes in species composition and life form groups. J. Veg. Sci. 6: 121-130.

Portes, V. L. 1996. Análisis de los cambios de usos del suelo en la sierra Zapalinamé. Municipios de Arteaga y Saltillo, Coah. Tesis de licenciatura. Universidad Autónoma Agraria Antonio Narro. Buenavista, Saltillo, Coah. 110 pp.

Portes, V. L. 2001. Evaluación del cambio de uso de suelo y del paisaje regional en la Sierra de Zapalinamé. Sociedades Rurales, Producción y Medio Ambiente 2(1): 41-51.

Puig, H., R. Bracho y V. J. Sosa. 1987. El bosque mesófilo de montaña: composición florística y estructura. In: Puig, H. y R. Bracho (eds.). El bosque mesófilo de montaña de Tamaulipas. Pub. Instituto de Ecología, A.C. México, D.F. pp. 55-79.

Quintana, P., M. González y N. Ramírez. 1992. Acorn removal, seedling survivorship and seedling growth of Quercus crispipilis in successional forests of the highlands of Chiapas, Mexico. Bull. Torrey Bot. Club 119: 6-18.

Ramírez, J. C. 1998. Un sistema de información geográfica para la identificación de los determinantes de la vegetación y usos del suelo en la Sierra de Zapalinamé, Coah., México. Tesis de licenciatura. Universidad Autónoma Agraria Antonio Narro. Buenavista, Saltillo, Coah. 103 pp.

Ramírez, N. 2003. Survival and growth of tree seedling in anthropogenically disturbed Mexican montane rain forests. J. Veg. Sci. 14: 881-890.

Ramírez, N., M. González y G. Williams. 2001. Anthropogenic disturbance and tree diversity in montane rain forest in Chiapas, México. For. Ecol. Manage. 154: 311-326.

Ruíz, H. C., J. Meave y J. L. Contreras. 2000. El bosque mesófilo de montaña de la región de Puerto Soledad (Oaxaca), México: análisis estructural. Bol. Soc. Bot. Mex. 65: 23-37.

Rzedowski, J. 1978. Vegetación de México. Ed. Limusa. México, D.F. 432 pp.

Saldaña, A., y E. J. Jardel. 1991. Regeneración natural del estrato arbóreo de bosques subtropicales de montaña en la Sierra de Manantlán, México: estudios preliminares. Biotam 3(3): 36-50.

Santiago, P. A. y E. J. Jardel. 1993. Composición y estructura del bosque mesófilo de montaña en la Sierra de Manantlán, Jalisco-Colima. Biotam 5(2): 13-26.

Smith, D. M. 1986. The practice of silviculture. John Wiley \& Sons, Inc., Nueva York. 527 pp. 
Spurr, S. H. y B. V. Barnes.1982. Ecología Forestal. AGT Editor. México, D.F 690 pp.

Valdés, T. V. y M. L. Aguilar. 1983. El género Quercus en las unidades fisonómico-florísticas del municipio de Santiago, N.L. México. Instituto Nacional de Investigaciones Forestales - Secretaría de Agricultura y Recursos Hidráulicos. México. Bol. Téc. No. 98. $94 \mathrm{pp}$.

Vásquez, G. J. A. y T. J. Givnish. 1998. Altitudinal gradients in tropical forest composition, structure, and diversity in the Sierra de Manantlán. Journ. Ecol. 86: 999-1020.

Vetaas, O. R. 1997. The effect of canopy disturbance on species richness in a central Himalayan oak forest. Plant Ecol. 132: 29-38.

Vetaas, O. R. 2000. The effect of environmental factor on the regeneration of Quercus semecarpifolia Sm. in central Himalaya, Nepal. Plant Ecol. 146(2): 137-144.

Villarreal, J. Á. 1994. Flora vascular de la Sierra de la Paila, Coahuila, México. Sida 16(1): $109-138$.

Villarreal, J. Á. 2001. Listados florísticos de México. XXIII Flora de Coahuila. Instituto de Biología. Universidad Nacional Autónoma de México. México, D.F. 137 pp.

Ward, J. 1963. Hierarchical grouping to optimize an objective function. Journ. Amer. Stat. Assoc. 58: 236-244.

White, P. S. y S. T. A. Pickett. 1985. Natural disturbance and patch dynamics: an introduction. In: Pickett, S. T. A. y P. S. White (eds.). The ecology of natural disturbance and patch dynamics. Academic Press. Orlando, Fl. pp. 3-13.

Wood, S., G. Harper, E. Muldavin y P. Neville. 1999. Vegetation map of the sierra del Carmen, U.S.A. and Mexico. U.S. Geological Service, National Wetlands Research Center, and the National Park Service. 143 pp.

Zar, J. H. 1984. Biostatistical analysis. 2a ed. Prentice Hall. Nueva York. 718 pp.

Zavala, F. 1995. Encinos hidalguenses. Universidad Autónoma de Chapingo. Chapingo, Edo. de México. 133 pp.

Zavala, F. y E. García M. 1997. Plántulas y rebrotes en la regeneración de encinos en la Sierra de Pachuca, Hidalgo. Agrociencia 31: 323-329.

Zavala, F. 2000. El fuego y la presencia de encinos. Ciencia Ergo Sum 7(3): 269-276. 


\title{
APÉNDICE
}

Lista florística de los bosques de encino de la Sierra de Zapalinamé

\section{PTERIDOPHYTA}

\begin{abstract}
Aspleniaceae
Asplenium monanthes L.

Asplenium resiliens Kunze
\end{abstract}

Equisetaceae

Equisetum hyemale L. var. affine (Engelm.) A.A. Eaton

Polypodiaceae

Pleopeltis guttata (Maxon) E.B. Andrews et Windham

Mildella intramarginalis (Kaulf. ex Link) Trevis. var. serratifolia (Hook. et Baker) C.C.

Hall et Lellinger

Pteridiaceae

Astrolepis sinuata (Lag. ex Sw.) D.M. Benham et Windham

Cheilanthes alabamensis (Buckley) Kunze

Cheilanthes eatoni Baker

Cheilanthes horridula Maxon

Cheilanthes pyramidalis Fée

Cheilanthes tomentosa Link

Notholaena aschenborniana Klotzsch

Notholaena incana C. Presl

Pellaea atropurpurea (L.) Link

Pellaea sagittata (Cav.) Link var. cordata (Cav.) A.F. Tryon

Pellaea ternifolia (Cav.) Link var. ternifolia

\section{PINOPHYTA}

\section{Cupressaceae}

Cupressus arizonica Greene

Juniperus flaccida Schltdl. var. flaccida

Pinaceae

Pinus cembroides Zucc.

Pinus greggii Engelm.

Pseudotsuga menziesii (Mirb.) Franco 


\section{MAGNOLIOPHYTA \\ DICOTYLEDONEAE (MAGNOLIOPSIDA)}

Acanthaceae

Dyschoriste schiedeana Kuntze

Anacardiaceae

Rhus aromatica Ait. var. trilobata (Nutt.) A. Gray

Rhus virens A. Gray var. virens

Apiaceae

Donnellsmithia ternata (S. Watson) Mathias et Constance

Eryngium hemsleyanum $\mathrm{H}$. Wolff

Apocynaceae

Mandevilla karwinskii (Müll. Arg.) Hemsl.

Asclepiadaceae

Cynanchum kunthii (Dcne.) Standl.

Asteraceae

Achillea millefolium L.

Ageratina calophylla (Blake) R.M. King et H. Rob.

Ageratina havanensis (Kunth) R.M. King et H. Rob.

Ageratina ligustrina (DC.) R.M. King et H. Rob.

Ageratina nesomii B.L. Turner

Ageratina oreithales (Greenm.) B.L. Turner

Ageratina scordoniodes (A. Gray) R.M. King et H. Rob.

Ageratina saltillensis (B.L. Rob.) R.M. King et H. Rob.

Ageratum corymbosum Zuccagni

Artemisia ludoviciana Nutt.

Aster carnerosanus S. Watson

Baccharis bigelovii A. Gray

Baccharis salicifolia (Ruiz et Pav.) Pers.

Bidens pilosa L.

Brickellia eupatorioides (L.) Shinners var. chlorolepis (Wooton et Standl.) B.L. Turner

Brickellia lemmonii A. Gray var. conduplicata (B.L. Rob.) B.L. Turner

Brickellia secundiflora (Lag.) A. Gray var. secundiflora

Brickellia veronicifolia (Kunth) A. Gray

Cirsium pringlei (S. Watson) Petr.

Chaetopappa parryi A. Gray

Chaptalia texana Greene

Dahlia coccinea Cav.

Dyssodia pinnata (Cav.) B.L. Rob. var. glabrescens Strother 
Erigeron basilobatus S.F. Blake

Erigeron pubescens Kunth

Fleischmannia pycnocephala (Lees.) R.M. King et H. Rob.

Gaillardia mexicana A. Gray

Gnaphalium roseum Kunth

Gnaphalium semiamplexicaule DC.

Gymnosperma glutinosum (Spreng.) Less.

Helianthella gypsophila B.L. Turner var. calcicola B.L. Turner

Helianthella mexicana A. Gray

Heliopsis parvifolia A. Gray

Heterotheca mucronata B.L. Turner

Hieracium crepidispermum Fr.

Koanophyllon solidaginifolium (A. Gray) R.M. King et H. Rob.

Lactuca graminifolia Michx.

Psacalium peltatum (Kunth) Cass. var. adenophorum S.F. Blake

Senecio loratifolius Greenm.

Senecio carnerensis Greenm.

Senecio coahuilensis Greenm.

Senecio madrensis A. Gray

Senecio richardsonii B.L. Turner

Smallanthus uvedalia (L.) Mack.

Solidago muelleri Standl.

Solidago velutina DC.

Stevia berlandieri A. Gray var. berlandieri

Stevia ovata Willd. var. ovata

Stevia porphyrea McVaugh

Stevia serrata Cav. var. serrata

Stevia salicifolia Cav. var. salicifolia

Stevia tomentosa Kunth

Tagetes lucida Cav.

Taraxacum officinale F.H. Wigg.

Verbesina coahuilensis A. Gray var. coahuilensis

Verbesina hypomalaca B.L. Rob. et Greenm. var. saltillensis B.L. Turner

Verbesina rothrockii B.L. Rob. et Greenm.

Vernonia greggii A. Gray var. greggii

Viguiera cordifolia A. Gray var. cordifolia

Viguiera dentata (Cav.) Spreng. var. dentata

Wedelia acapulcensis Kunth var. hispida (Kunth) Strother

Berberidaceae

Berberis eutriphylla (Fedde) C.H. Mull.

Berberis trifoliolata Moric. var. glauca (I.M. Johnst.) M.C. Johnst. 
Betulaceae

Ostrya virginiana (Mill.) K. Koch

Boraginaceae

Lithospermum viride Greene

Brassicaceae

Asta schaffneri (S. Watson) O.E. Schulz var. pringlei (O.E. Schulz) Rollins

Cardamine macrocarpa Brandegee var. macrocarpa

Erysimum capitatum (Douglas) Greene

Lesquerella argyraea (A. Gray) S. Watson ssp. diffusa (Rollins) Rollins et E.A. Shaw

Schoenocrambe linearifolia (A. Gray) Rollins

Thelypodium longipes (Rollins) Rollins

Buddlejaceae

Buddleja cordata Kunth ssp. tomentella (Standl.) E.M. Norman

Cactaceae

Opuntia robusta H.L. Wendl. var. robusta

Opuntia stenopetala Engelm. var. stenopetala

Caprifoliaceae

Lonicera pilosa (Kunth) Willd.

Symphoricarpos microphyllus Kunth

Caryophyllaceae

Arenaria lanuginosa (Michx.) Rohrb.

Drymaria glandulosa C. Presl var. glandulosa

Silene laciniata Cav.

Celastraceae

Paxistima myrsinites (Pursh) Raf. ssp. mexicana A.M. Navarro et W.H. Black.

Cistaceae

Helianthemum glomeratum (Lag.) Dunal

Convolvulaceae

Ipomoea hederifolia L.

Cornaceae

Cornus stolonifera Michx.

Crassulaceae

Echeveria strictiflora A. Gray

Sedum wrightii A. Gray ssp. priscum R.T. Clausen 
Cucurbitaceae

Sicyos angulatus L.

Ericaceae

Arbutus xalapensis Kunth

Arctostaphylos pungens Kunth

Euphorbiaceae

Acalypha lindheimeri Müll. Arg.

Euphorbia brachycera Engelm. var. brachycera

Euphorbia dentata Michx.

Tragia ramosa Torr.

Fabaceae

Astragalus greggii S. Watson

Astragalus legionensis Barneby

Cercis canadensis L. var. mexicana (Rose) M. Hopkins

Cologania angustifolia Kunth

Cologania pallida Rose

Dalea frutescens A. Gray

Dalea radicans $\mathrm{S}$. Watson

Desmodium grahamii A. Gray

Desmodium lindheimeri Vail

Desmodium psilophyllum Schltdl.

Lathyrus parvifolius S. Watson

Phaseolus filiformis Benth.

Medicago polymorpha L. var. vulgaris (Benth.) Shinners

Mimosa aculeaticarpa Ortega

Nissolia platycalyx S. Watson

Vicia ludoviciana Nutt. ssp. ludoviciana

Fagaceae

Quercus greggii (A. DC.) Trel.

Quercus grisea Liebm.

Quercus hypoxantha Trel.

Quercus laceyi Small

Quercus laeta Liebm.

Quercus mexicana Kunth

Quercus pringlei Seemen

Quercus rugosa Née

Quercus saltillensis Trel.

Quercus sideroxyla Kunth

Quercus striatula Trel. 
Garryaceae

Garrya glaberrima Wangerin

Garrya ovata Benth. ssp. ovata

Geraniaceae

Geranium crenatifolium H.E. Moore

Geranium seemannii Peyr.

Hydrophyllaceae

Nama dichotomum (Ruiz et Pav.) Choisy var. dichotomum

Hypericaceae

Hypericum punctatum Lam.

Lamiaceae

Hedeoma costatum A. Gray var. costatum

Stachys bigelovii A. Gray

Salvia coahuilensis Fernald

Salvia regla Cav.

Salvia glechomifolia Kunth

Salvia greggii A. Gray

Salvia roemeriana Scheele

Lauraceae

Litsea parvifolia (Hemsl.) Mez

Linaceae

Linum schiedeanum Schltdl. et Cham.

Lythraceae

Cuphea aequipetela Cav.

Cuphea cyanea DC.

Nyctaginaceae

Mirabilis oblongifolia (A. Gray) Heimerl

Oleaceae

Fraxinus cuspidata Torr.

Onagraceae

Oenothera rosea Aiton

Orobanchaceae

Conopholis alpina Liebm. var. mexicana (A. Gray) R. R. Haynes 
Oxalidaceae

Oxalis latifolia Kunth

Oxalis drummondii A. Gray

Polemoniaceae

Loeselia scariosa (M. Martens et Galeotti) Walp.

Polygalaceae

Polygala lindheimeri A. Gray var. eucosma (S.F. Blake) T. Wendt

Polygonaceae

Eriogonum atrorubens Engelm. var. auritulum W.J. Hess et Reveal

Pyrolaceae

Chimaphila umbellata (L.) Nutt.

Rhamnaceae

Ceanothus caeruleus Lag.

Rhamnus betulifolia Greene

Ranunculaceae

Clematis pitcheri Torr. et A. Gray

Thalictrum grandidentatum S. Watson

Rosaceae

Alchemilla vulcanica Cham. et Schltdl.

Amelanchier denticulata (Kunth) K. Koch.

Cercocarpus fothergilloides Kunth

Cercocarpus montanus Raf.

Crataegus baroussana Eggl. var. baroussana

Fragaria virginiana Duchesne var. glauca S. Watson

Holodiscus discolor (Pursh.) Maxim. var. dumosus (Nutt.) Maxim.

Lindleya mespiloides Kunth

Prunus mexicana S. Watson

Prunus serotina Ehrh. ssp. virens (Wooton et Standl.) McVaugh

Purshia plicata (D. Don) Henrickson

Rubus trivialis Michx.

Rubiaceae

Bouvardia ternifolia (Cav.) Schltdl.

Galium uncinulatum A. Gray

Hedyotis nigricans (Lam.) Fosberg. var. rigidiuscula (A. Gray) Shinners

Relbunium microphyllum (A. Gray) Hemsl. 
Salicaceae

Populus tremuloides Michx.

Salix lasiolepis Benth.

Salix paradoxa Kunth

Sapotaceae

Bumelia lanuginosa (Michx.) Pers.

Scrophulariaceae

Castilleja scorzonerifolia Kunth

Penstemon barbatus (Cav.) Roth. var. barbatus

Penstemon campanulatus (Cav.) Willd. ssp. campanulatus

Seymeria decurva Benth.

Solanaceae

Nectouxia formosa Kunth

Nierembergia angustifolia Kunth

Physalis hederaefolia A. Gray var. hederaefolia

Physalis viscosa L. var. cinerascens (Dunal) Waterf.

Solanum nigrum L.

Solanum verrucosum Schltdl.

Verbenaceae

Verbena neomexicana (A. Gray) Small var. hirtella L. M. Perry

Violaceae

Viola sororia Willd.

Viscaceae

Phoradendron lanceolatum Engelm.

Phoradendron tomentosum (DC.) Engelm.

\section{MONOCOTILEDONEAE (LILIOPSIDA)}

Agavaceae

Agave gentryi B. Ullrich

Nolina cespitifera Trel.

Yucca carnerosana (Trel.) McKelvey

Bromeliaceae

Tillandsia erubescens Schltdl.

Tillandsia recurvata $\mathrm{L}$. 
Commelinaceae

Aneilema linearis (Benth.) Woodson

Commelina dianthifolia Delile

Commelina diffusa Burm. f.

Commelina erecta L. var. angustifolia (Michx.) Fernald

Cyperaceae

Carex schiedeana Kunze

Cyperus odoratus L.

Iridaceae

Sisyrinchium scabrum Cham. et Schtdl.

Liliaceae

Echeandia flavescens (Schult. et Schult.) Cruden

Schoenocaulon macrocarpum Brinker

Orchidaceae

Corallorhiza maculata (Raf.) Raf.

Goodyera oblongifolia Raf.

Govenia liliacea (La Llave et Lex.) Lindl.

Malaxis brachystachya (Rchb.) Kuntze

Malaxis soulei L.O. Williams

Poaceae

Aristida schiedeana Trin. et Rupr. var. schiedeana

Bouteloua curtipendula (Michx.) Torr. var. curtipendula

Bouteloua dactyloides (Nutt.) Columbus

Bouteloua hirsuta Lag.

Brachypodium mexicanum (Roem. et Schult.) Link var. mexicanum

Bromus anomalus Rupr. ex E. Fourn.

Bromus carinatus Hook. et Arn.

Elymus arizonicus (Scribn. et Smith) Gould

Eragrostis intermedia Hitchc.

Koeleria macrantha (Ledeb.) Schult.

Leptochloa dubia (Kunth) Nees

Lycurus phleoides Kunth

Panicum bulbosum Kunth

Piptochaetium fimbriatum (Kunth) Hitchc.

Polypogon viridis (Gouan) Breistr.

Muhlenbergia dubia Hemsl.

Muhlenbergia emersleyi Vasey

Muhlenbergia glauca (Nees) Mez

Muhlenbergia setifolia Vasey 
Acta Botanica Mexicana 86: 71-108 (2009)

Nassella leucotricha (Trin. et Rupr.) Barkw.

Nassella mucronata (Kunth) R.W. Pohl

Schizachyrium scoparium (Michx.) Nash var. scoparium

Sorghastrum brunneum Swallen

Stipa eminens Cav.

Trisetum deyeuxioides (Kunth) Kunth

Trisetum spicatum (L.) K. Richt.

Trisetum viride (Kunth) Kunth

Smilacaceae

Smilax bona-nox L. 